\title{
DIE GESETZGEBUNG IN DER VOLKSREPUBLIK CHINA
}

\author{
Von OsKar WeggeL
}

\section{A. Das Gesetzgebungsverfahren in der Theorie}

Theoretisch ist die Zuständigkeit für die Gesetzgebung in der Volksrepublik China klar definiert. Sie steht gemäß Art. 22 der Verfassung vom 20. 9.1954 ausschließlich dem „Nationalen Volkskongreß“ $\mathrm{zu}^{1}$. Das Gesetzgebungsverfahren verläuft in drei Takten:

a) Die Gesetzesinitiative liegt beim Staatsrat ${ }^{2}$. Auch das „Vorschlagsrecht" der obersten Staatskonferenz ${ }^{3}$ dürfte die Initiativbefugnis mit umfassen ${ }^{4}$. Schließlich liegt es nahe, daß die Nationalversammlung auch aus ihrer eigenen Mitte heraus initiativ tätig werden kann, obwohl davon in der Verfassung nicht ausdrücklich die Rede ist.

b) Die Nationalversammlung beschließt mit einfacher Stimmenmehrheit ${ }^{5}$ über die Gesetze ${ }^{6}$, die sodann

c) vom Vorsitzenden der Volksrepublik China, also vom Staatspräsidenten, ve r k ü n d e t werden?.

Mit „Gesetzen" sind hier nur solche f o r m e $1 \mathrm{l}$ e $\mathrm{r}$ Natur gemeint.

$\mathrm{M}$ a t e ri elle s Recht kann auch von anderen Staatsorganen, z. B. vom Ständigen Ausschuß des Nationalen Volkskongresses und vom Staatsrat erlassen werden ${ }^{8}$. Der Ständige Ausschuß ist insbesondere dazu ermächtigt, „Gesetze auszulegen “9, „Erlasse herauszugeben"10 sowie "Verordnungen und Verfügungen des Staatsrats aufzuheben, die im Widerspruch zur Verfassung, zu den Gesetzen oder zu Erlassen stehen"11. Der Staatsrat hat ebenfalls das Recht, „Verordnungen und Verfügungen zu erlassen "12, und selbst die Minister sind befugt, aufgrund der Gesetze und Erlasse sowie aufgrund der Verordnungen und Verfügungen des Staatsrats "Anordnungen und Instruktionen" herauszugeben ${ }^{13}$, womit allerdings im wesentlichen innerdienstliche Verfügungen, also Anordnungen im „besonderen Gewaltverhältnis" gemeint sein dürften. Gewisse Anordnungsbefugnisse stehen auch dem Staatspräsidenten $\mathbf{z u}^{14}$.

Außerdem sind noch die „örtlichen Volkskongresse“ ermächtigt, Beschlüsse zu fassen und $\mathrm{zu}$ verkündigen ${ }^{15}$.

Mit diesen Präliminarien ist die rein juristische Betrachtungsweise freilich bereits am Ende. Besteht doch — ähnlich wie in anderen kommunistischen Ländern — das

1 jen-min tai-piao ta hui.

2 kuo-wu-yüan.

3 tsui kao kuo-wu hui-i: sie hat die Aufgabe, eng mit dem Staatspräsidenten zusammenzuarbeiten und ist insofern dessen Sprachrohr (Art. 43 Verf.).

443 Abs. 3 Verf.

5 Art. 27 Ziff. 2,29 Abs. 2 Verf.

6 fa-lü.

7 fa-lü. 40 Verf.

8 Vgl. Hermann Jensen „Die Verfassung der VRC mit besonderer Berücksichtigung der Verfassungsgrundsätze und ihrer Anwendung in der Verfassungswirklichkeit ${ }^{\alpha}$, Diss. Marburg 1964, S. 125.

9 Art. 31 Ziff. 3 Verf.

10 Art. 31 Ziff. 4 (Erlaß = Fa-ling)

11 Art. 31 Ziff. 6 Verf.

12 Art. 49 Ziff. 1 Verf. (Verordnungen und Verfassungen = chüeh-i ho chih-shih)

13 Art. 51 Verf. (Anordnungen u. Instruktionen = ming-ling ho chih-shih).

14 Art. 40 Verf.

15 Art. 60 Verf. („Beschlüsse ${ }^{\alpha}=$ chüeh-i). In diesem Zusammenhang sei der - völlig unergiebige Aufsatz von William T. Wright "Gesetzgebung und Rechtsprechung in der VRC (Osteuropa-Recht 1958 S. 187) wenigstens erwähnt. 
Dilemma der formellen Verfassung gerade darin, daß sie, wenn man die $t$ a $t$ s ächlich e Normsetzungstätigkeit als Maßstab nimmt, praktisch nie so recht gegolten hat.

Der Komplex der Gesetzgebung läßt sich überhaupt nur in den Griff bekommen, wenn man ihn nicht aus der gesamtgesellschaftlichen Wirklichkeit herauslöst und isoliert betrachtet, sondern ihn als integrierenden Bestandteil der Sozialordnung im kommunistischen China gelten läßt. Diese Zusammenschau hat freilich zur Folge, daß sämtliche Implikationen des gesellschaftlichen Geschehens in die juristische Betrachtungsweise mit einbezogen werden müssen. Gerade aus diesem Grunde folgt die Abhandlung mehr ideologischen und soziologischen Kategorien als juristischen.

\section{B. Die Praxis}

\section{Bestandsaufnahme}

\section{Die Geschichte der Gesetzgebung in der Volksrepublik China}

$\mathrm{Da}$ die Legislative keine institutionelle Sonderexistenz führt, folgt ihre Geschichte ganz der allgemeinen politischen Praxis in der Volksrepublik, die manchmal institutionsfreundliche Strömungen zeigte, im Laufe der Jahre dem Recht und rechtlichen Einrichtungen jedoch immer mehr mit Feindschaft und Mißtrauen begegnete. Nimmt man diese grundsätzliche Haltung als Kriterium, so ergeben sich fünf Perioden der Gesetzgebungspolitik ${ }^{\mathbf{1 6}}$ :

a) Phase I (1949-1952) stand noch ganz im Zeichen des Bürgerkrieges und trug das Gepräge der Pionierzeit. Auseinandersetzungen mit den Überresten der Kuomintang in den administrativen, erzieherischen, landwirtschaftlichen und industriellen Einheiten wirbelten die überkommene Gesellschaftsstruktur durcheinander. Nichts war deshalb weniger zu gebrauchen als Gesetze, die ja gerade zur Stabilisierung eines im Umbruch begriffenen Zustandes geführt hätten. Verständlicherweise wetterte damals die Volkszeitung gegen „einige Leute, die ohne Rücksicht auf die gegenwärtigen praktischen Bedingungen ... auf den Erlaß einer vollständigen Gesetzessammlung drängen ${ }^{17}$."

b) Phase II (1953-1957) brachte dann eine Wendung um $180^{\circ}$. Insbesondere das Jahr 1954 bedeutete, wie unten noch näher auszuführen ist ${ }^{18}$, den Höhepunkt der administrativen Verfestigung und Institutionalisierung. Damals hat sich die Entwicklung wohl am meisten von den maoistischen Vorstellungen wegentwickelt. Kein Wunder, daß der Ruf „nach einigermaßen vollständigen Grundstatuten . . ., wie einem Strafgesetzbuch, einem Zivilgesetzbuch, nach Prozeßordnungen, nach einem Arbeitsgesetz u. dgl. ..." besonders laut wurde ${ }^{19}$.

16 Arthur Stahnke ( $T$ The Background and Evolution of Party Policy on the Drafting of Legal Codes in Communist China in "The American Journal of Comparative Law “ Band XV (1966/67, S. 507), gliedert die Gesetzgebungsgeschichte in drei Perioden, wobei er die dritte Phase mit dem Jahr 1957 einsetzen läßt. Da Stahnkes Arbeit vor oder aber kurz nach Beginn der Kulturrevolution geschrieben wurde, konnte er die weiteren Veränderungen noch nicht überblicken. Zumindest aber hätte er dem Einschnitt nach 1961 Rechnung tragen müssen.

17 Pekinger Volkszeitung (fortan „VZ“) v. 5. 9. 1951, zit. bei Stahnke a. a. O. 507.

18 Unten B II I. "Completely smash the Feudal, Capitalist and Revisionist Legal Systems in "Survey of China Mainland "Magazines“ (hinfort SCMM) Nr. 625 (Sept. 1968), S. 23 (nach: Canton Fan P'eng-Lo hei-hsien Nr. 2 Juli 1968). 
c) Phase III (1957-1960): Auf die "rechtsabweichlerische“ Kritik zahlreicher Juristen am Rechtssystem der Volksrepublik China während der „Hundert-BlumenPeriode ${ }^{20}$ antwortete die Partei mit einer scharfen Abwendung vom bisherigen Gesetzgebungskurs. „... im gegenwärtigen Zeitpunkt darf unser Land nur ein paar provisorische Vorschriften ... erlassen, welche im wesentlichen allgemeine Programme und Grundsätze enthalten ... Wir sind keine Theoretiker, die glauben, daß Gesetze alles schaffen können ... In einer Periode, in der unsere Gesellschaft ihr Gesicht verändert, ..., ist es nicht nötig, ..., alle Maßnahmen der Partei in Gesetzesvorschriften umzumünzen ... “21.

d) Phase IV (1960-1966): Nach der mißlungenen Politik des "Großen Sprungs“ und der sich anschließenden Wirtschaftsmisere in den Jahren von 1959 bis 1961 geriet die "Linie des Vorsitzenden Mao" für einige Zeit ins Hintertreffen. Es war dies eine Zeit, in der Liu Shao-ch'i - gewarnt von den vorausgegangenen Ereignissen - für eine gemäßigtere Politik plädierte und dabei auch in der Partei zustimmendes Echo fand. Diese allgemeine Mäßigung der Politik schlug sich auch auf das Recht nieder: P'eng $\mathrm{Chen}^{22}$ forderte z. B. stärkere Unabhängigkeit der Gerichte und wollte vor allem der Partei verbieten, sich in die Justiz einzumischen ${ }^{23}$. Er beklagte den verbreiteten Mangel an Rechtskenntnissen und forderte, daß „wir uns zwei oder drei Jahre ... mit chinesischen und ausländischen Büchern über juristische Fragen beschäftigen " müßten ${ }^{24}$. Zu allem Überfluß wurde auch die Forderung laut, den „Sechs Gesetzbüchern der Kuomintang“ zu einer Renaissance $\mathrm{zu}$ verhelfen ${ }^{25}$.

e) Phase V (nach 1966): All diese Forderungen gerieten nach 1966 abermals in Vergessenheit. Mit der Kulturrevolution nämlich, die der Massenlinie wieder voll zum Durchbruch verhelfen sollte und die deshalb insbesondere die etablierten Apparate von Partei und Verwaltung zerschlug, begann eine Periode, in der die Animosität gegenüber Recht und rechtlich verfestigten Institutionen eine nie gekannte Intensität erreichte. Zumindest im zweiten Halbjahr des Jahres 1966 und in den ersten Monaten des Jahres 1967 hatte die legale Herrschaft zeitweilig fast vollständig einem charismatisch legitimierten System Platz zu machen ${ }^{26}$.

Der ständige Wechsel in der Gesetzgebungspolitik, wie er in dieser Übersicht zutage tritt, läßt die Frage aufkommen, ob ein so umfangreiches Gebiet sich überhaupt adäquat untersuchen läßt. Vielleicht vermag folgende Überlegung eine Antwort auf diese Frage zu geben:

\section{Vergleich zweier polarer „Legislaturperioden“}

In einer Analyse, die es sich zur Aufgabe gesetzt hat, die wesentlichen Elemente der Gesetzgebung in der Volksrepublik China herauszuarbeiten, wäre es wenig sinnvoll, sämtliche Gesetze, Verordnungen und dgl. auszuwählen, die im Ver-

\footnotetext{
$20 \mathrm{Vgl}$. Roderick McFarqhar "The Hundred Flowers Campaign and the Chinese Intellectuals", 2. Aufl. NY 1960, S. $114 \mathrm{ff}$.

21 Chung-hua jen-min kung-ho-kuo min-fa chi-pen wen-t'i („Grundprobleme des Bürgerl. Rechts der VRC“), Peking 1958, S. 12.

22 OB von Peking, 1. Vors. des Pekinger Parteiausschusses, Mitglied des Politbüros.

23 Vgl. SCMM Nr. 625 'FN 19), S. 27

24 Ib. 27.

25 Eine Forderung, die angeblich vor allem vom Vorsitzenden des Volksgerichtshofs von Canton, Kao K'un-feng erhoben wurde, ib. $27 \mathrm{f}$.

26 Vgl. dazu unter B II I.
} 
laufe von 20 Jahren publiziert wurden ${ }^{27}$. Der oben gegebene geschichtliche Überblick über die fünf verschiedenen Gesetzgebungsphasen der Volksrepublik sollte genügen, die Entwicklung wenigstens in großen Zügen zu charakterisieren.

Worauf es im vorliegenden Zusammenhang vor allem ankommt, ist eine Konfrontation von Extremzuständen, die die beiden gleichsam polaren Möglichkeiten der chinesischen "Legislative" aufzuzeigen vermögen. Das Auf und Ab der geschichtlichen Entwicklung macht es einem leicht, solche Fixpunkte aufzufinden. Vor allem sind es die "Legislaturperioden" von 1954/55 einerseits und von 1966/67 andererseits, die wohl am weitesten voneinander entfernt sind und bei denen das Pendel der Gesetzgebung am stärksten ausgeschlagen hat.

Pauschal ausgedrückt versinnbildlicht die Phase 1954/55, die ja ganz im Zeichen des sowjetischen Vorbilds steht, die „westliche" Periode der chinesischen Gesetzgebung, während die Normen von 1966/67 ganz vom Maoismus geprägt sind.

Beide Pole zeigen etwa den Rahmen auf, innerhalb dessen auch die künftige Strategie der "Legislative" funktionieren wird und verdienen gerade deshalb besondere Aufmerksamkeit.

Der Vergleich zwischen beiden Perioden soll vor allem in drei Stücken durchgeführt werden, wobei die nachfolgenden Tabellen als Materialsammlung dienen mögen. Es gilt zunächst, die formalen Normtitel zu vergleichen (Tabelle I); anschließend werden die "Gesetzgebungsorgane" (Tabelle II) und schließlich die Gesetzgebungsmaterien (Tabelle III) einander gegenübergestellt. Die in beiden Perioden recht unterschiedlich gehandhabte "Gesetzgebungs"-Technik ist anschließend anhand einiger organisatorischer Regelungen zu verdeutlichen. Ausführungen über die „Neuesten Weisungen des Vorsitzenden“ sollen die Gegenüberstellung abrunden. Als Vergleichsgrundlage dienen für 1954/55 die in der Amtlichen Chinesischen Gesetzessammlung veröffentlichten Normen ${ }^{28}$ und für 1966/67 die vom Union Research Institute/Hongkong herausgegebene „Dokumentensammlung"29.

27 Die Gesetzgebung der Volksrepublik China wurde in zwei amtlichen Sammlungen veröffentlicht:

a) chung-yang jen-min cheng-fu fa-ling hui-pien („Sammlung der Gesetze und Verordnungen der Zentralen Volksregierung “). Diese Sammlung umfaßt die Periode von 1949 bis 1954, also sozusagen die vorkonstitutionelle Gesetzgebung.

b) Ferner existiert die im vorliegenden Zusammenhang besonders wichtige chung-hua jen-min kung-hokuo fa-kui hui-pien („Sammlung der Rechtsbestimmungen der Chinesischen Volksrepublik ). Diese Sammlung umfaßt den Zeitraum von 1954 bis 1963 , also sämtliche Rechtsnormen, die nach Erlaß der Verfassung verkündet wurden. Jeder Band ist mit einer fortlaufenden Nummer bezeichnet, mit dem Ergebnis, daß es insgesamt 13 Bände gibt.

Ergebnis, daß es insgesamt 13 Bände gibt.
c) Für die „Gesetzgebung “ nach 1963 ist auf zwei Quellen zurückzugreifen:

a) jen-min tai-piao ta hui ch'ang-wu wei-yüan-hui kung-pao („Bekanntmachungen des Ständigen Ausschusses beim Nationalen Volkskongreß“). Diese „Bekanntmachunge ${ }^{\alpha}$ wurden bis 1966 publiziert. Seitdem fehlt jede Spur.

bb) Ferner ist hier wichtig das kuo-wu-yüan kung-pao („Bekanntmachungen des Staatsrats“).

$\mathrm{Zu}$ bemerken wäre, daß die letzten drei Bände der fa-kui und die Bekanntmachungen des Ständigen Ausschusses des Nationalen Volkskongresses sowie des Staatsrates nur bruchstückweise ins Ausland gelangt sind. Das Amtsblatt des Ständigen Ausschusses des Nationalen Volkskongresses z. B. ist in Deutschland für die Jahre 1965 und 1966 nur in der "Seminarabteilung für Ostrechtsforschung der Universität Hamburg " vorhanden (die letzte Ausgabe, die in der amerikanischen Library of Congress verzeichnet ist, reicht demgegenüber nur bis Sept. 1965; Näheres vgl. „Osteuroparecht ${ }^{\star}{ }_{1969}$, S. 249). Einige Nummern des Amtsblattes des Staatsrats liegen im Institut für Asienkunde, Hamburg, auf Mikrofilm vor.

28 chung-hua jen-min kung-ho-kuo fa-kui hui-pien, Bd. 1 und 2, beide Peking 1956.

29 „CCP Documents of the Great Proletarian Cultural Revolution 1966-67“ Hongkong 1968 (fortan als ${ }_{n} \mathrm{DB}^{\alpha}=$ „Dokumentenband “ abgekürzt). Diese Sammlung erfaßt zwar nur einen Teil der 1966/67 herausgegebenen rd. 800 Dokumente: Das Union Research Institute war aber aufgrund seiner umfassenden Materialsammlung und der Fachkenntnis seiner zahlreichen Mitarbeiter in der Lage, eine repräsentative Auswahl zu treffen. 


\section{Tabelle I}

Gegenüberstellung der Normen,titel“

(nach Häufigkeit angeordnet)

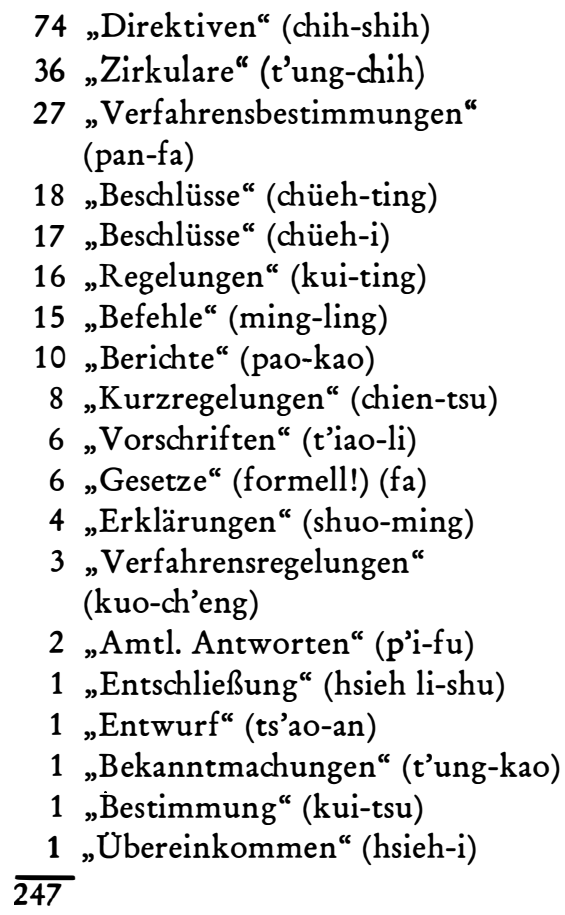

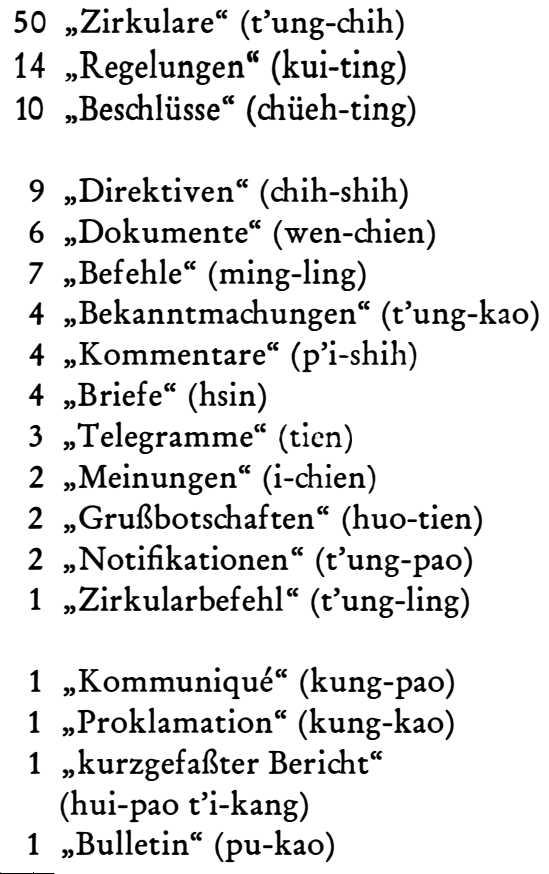


Tabelle II

Gegenüberstellung der „Gesetzgebenden“ Organe

$1954 / 55$

(nach Häufigkeit angeordnet)

1. Staatsrat und seine Minister . 190 (StR: 88, Min: 102)

2. Nationaler Volkskongreß, Ständiger Ausschuß . . . . . 28

3. Nationaler Volkskongreß ... 24

4. Staatsbank ......... 2

5. Staatspräsident ....... 1

6. Zentralkomitee

( + einmal ZK in Verbindung

mit Staatsrat: 1) . . . . . $\frac{1}{247}$

\section{Abkürzungen:}

$\mathrm{ZK}=$ Zentralkomitee der kommunistischen Partei Chinas

ZKMA $=$ Militärausschuß beim Zentralkomitee

ZKRA $=$ Revolutionsausschuß beim Zentralkomitee

StR $=$ Staatsrat

$\mathrm{VBA}=$ Volksbefreiungsarmee
1. Zentralkomitee allein ..... . 51

2. ZK + ZKMA + ZKRA + StR 17

3. ZKMA ......... 16

$4 . Z K+S t R \ldots \ldots 15$

5. $\mathrm{ZK}+\mathrm{StR}+\mathrm{ZKMA} \ldots \ldots 5$

6. StR ........... 5

7. StR + ZKMA ....... 4

8. ZKRA .............. 3

9. ZK + ZKRA ....... 2

10. $\mathrm{ZK}+\mathrm{ZKMA}+\mathrm{ZKRA}$

+ VBA-Kulturrevol. Gruppe 2

11. ZK + ZKMA ....... 1

12. „Gruppe der Fünf“ . . . . . . 1 


\section{Tabelle III}

\section{Gegenüberstellung der „Gesetzes“-Materien}

$\begin{array}{lrr}\text { „Allgemein-revolutionäres" Recht } & 4 & 81 \\ \text { Organrecht } & 36 & \\ \text { Inneres } & 16 & \\ \text { Außeres } & 2 & 16 \\ \text { Militär } & 9 & 2 \\ \text { Sicherheit etc. } & 16 & 2 \\ \text { Wirtschaft } & 5 & \\ \text { Aufbau } & 5 & \\ \text { Finanzen, Währung } & 24 & \\ \text { Ernährung } & 11 & 3 \\ \text { Handel } & 8 & \\ \text { Industrie, Verkehr } & 10 & 2 \\ \text { Landwirtschaft, Forsten } & 23 & 7 \\ \text { Arbeit } & 25 & \\ \text { Kultur, Wissenschaft } & 14 & \\ \text { Erziehung } & 22 & \\ \text { Gesundheit, Sport } & 5 & 3 \\ \text { Minoritäten } & 6 & 122 \\ \text { Auslandschinesen } & 2 & \\ \text { Dienstrecht } & 4 & \\ & 247 & \end{array}$

\section{a) Auswertung der Tabelle I}

Wie schon ein erster Blick auf die Tabelle zeigt, weist die Nomenklatur der Titel eine geradezu inflationäre Tendenz auf. Aus dem Jahre 1954/55 sind nicht weniger als 19 Arten zu ermitteln und aus dem Jahre 1966/67 immerhin auch 18 verschiedene Kategorien. Es fällt auf, daß vor allem „Direktiven“, „Zirkulare“, „Regelungen“, „Beschlüsse“ und „Befehle“ ganz entschieden die Skala der Normtitel anführen. An formellen Gesetzen lassen sich in der Periode I nur 7, in der Periode II dagegen überhaupt keines entdecken ${ }^{30}$.

30 Neben dem „Gesetz über den Militärdienst ${ }^{\alpha}$ vom 30. 7. 1955 wurden noch fünf Organstatuten als formelle Gesetze erlassen, nämlich die Organisationsesetze für den Nationalen Volkskongreß (vom 20. 9. 1954), für den Staatsrat (vom 21. 9. 1954), für den Volksgerichtshof (vom 21. 9. 1954), für die 20. 9. 1954), für den Staatsrat (vom 21. 9. 1954), für den Volksgerichtshof (vom 21. 9. 1954), für die
Volksstaatsanwaltschaft (vom 21. 9. 1954) und für die lokalen Volkskongresse und Volksräte (vom 21. 9. 1954). Die Gesetze über den Staatsrat sowie über die lokalen Volkskongresse und Volksräte wurden ein Jahr später, nämlich am 30. Juli 1955, durch formelles Gesetz geringfügig abgeändert.

Hinzuzufügen wäre noch, daß $n$ a $c h$ dem Militärdienstgesetz kein formelles Gesetz mehr erlassen wurde. Insoweit beschränkt sich die Angabe der formellen Gesetze hier also nicht nur auf die Periode 1954/55!

Erstaunlicherweise sind die vorkonstitutionellen formellen Gesetze viel reichlicher gesät als die nachkonstitutionellen. Eine ausgezeichnete Auswahl dieser vorkonstitutionellen Gesetze findet sich bei Albert P. Blaustein: Fundamental Legal Documents of Communist China „South Hackensack, New Jersey, 1962. Nach dem Beschluß des Nationalen Volkskongresses über den Fortbestand der vorkonstitutionellen Gesetze vom 26. 9. 1954 gelten sämtliche seit dem 1. Oktober 1949 durch die Volksrepublik erlassenen Gesetze, ferner alle von der Volksregierung gefaßten Beschlüsse und alle von der Volksregierung bestätigten Rechtsnormen insoweit weiter, als sie der Verfassung von 1954 nicht widersprechen (vgl. den entsprechenden Beschluß in Bd. I der chung-hua jen-min kung-ho-kuo fa-kui hui-pien, S. 110). 
Es erscheint wenig sinnvoll, dieses bunte Kaleidoskop von Normen unter deutsche Begriffe wie "Gesetze“, „Verordnungen“, „Satzungen“, „Beschlüsse“, „Verfügungen“ usw. zu subsumieren; denn die so harmlos klingenden „Briefe“, wie sie etwa in der Periode von 1966/67 auftauchten, besitzen oft eher den Charakter einer Rechtsnorm ${ }^{31}$ als die soviel bedeutsamer scheinenden „Beschlüsse“, die teilweise nur eine Art Empfehlung oder Information zum Inhalt haben ${ }^{32}$. Auch gehen Meinungsäußerungen, Empfehlungen, generelle Anweisungen und Kampfaufrufe allgemeinster Art oft in einem einzigen Dokument so kunterbunt durcheinander, $\mathrm{da}$ nur eine genaue Abwägung, welchen Elementen das Übergewicht zukommt, $\mathrm{zu}$ einer einigermaßen adäquaten Zuordnung führen könnte ${ }^{33}$. Hinzu kommt, daß die Terminologie selbst nicht konsequent angewandt wird: Ein Außenstehender ist beispielsweise geneigt, unter „Bekanntmachung“ eine öffentlich anzuschlagende und unter "Zirkular" eine für den Umlauf bestimmte Anordnung zu verstehen. In der Praxis geschieht es aber oft gerade umgekehrt ${ }^{34}$.

Müßig wäre es, hier zu entscheiden, ob solchen „Anweisungen“ oder wie immer der Titel lauten möge, überhaupt noch der Charakter einer Rechtsnorm zukommt. Eine solche Entscheidung erforderte präzise Definitionen des Rechtsbegriffs und würde damit ein Problem aufwerfen, das in der Rechtsphilosophie ebenso häufig ungelöst blieb, wie es in Angriff genommen wurde ${ }^{\mathbf{3 5}}$.

Gewisse Mindestvoraussetzungen für die Rechtssatzqualität, vor allem solche, die eine Rechtsnorm von einer bloßen Verfügung abheben, also den Charakter genereller Verbindlichkeit tragen, sind jedenfalls gegeben. Das ZK oder andere Instan-

$31 \mathrm{Vgl}$. etwa den Brief des $\mathrm{ZK}$, an die armen und unteren Mittelbauern sowie an die Kader aller Ebenen in den Volkskommunen des ganzen Landes" vom 20. 2. 1967 (DB 331 ff.), wo die Behandlung der Kader in ländlichen Gebieten generell verbindlich geregelt wird.

32 Vgl. z. B. den „Beschluß ${ }^{\alpha}$ des ZK, des Staatsrats und des Militärausschusses beim ZK, den ChechiangZwischenfall betreffend, vom 30. 11. 1967 (DB $221 \mathrm{ff}$. insbesondere Abs. 4 und 5).

33 Vgl. z. B. den Beschluß in FN 32.

34 DB S. iii.

35 Um die Schwierigkeit des Unternehmens anzudeuten, seien hier wenigstens einige Begriffsdefinitionen wiedergegeben:

1. Eine formale Definition stammt von Gustav Radbruch ( $R$ Recht ist die Wirklichkeit, die den Sinn hat, dem Rechtswerte, der Rechtsidee zu dienen ${ }^{\alpha}$ in "Rechtsphilosophie ${ }^{\star}$, hrsg. v. Erik Wolff, Stuttgart 1956, S. 123; „Vorschule der Rechtsphilosophie“ Göttingen 1959, 2. Aufl., S. 34). Diese Definition ist vor allem in Hinblick auf rechtswidrige Rechtsnormen entwickelt worden und muß im vorliegenden Fall schon deshalb als Abgrenzungskriterium versagen.

2. Materielle Definitionen.

a) Die häufigsten vier Kriterien für das Recht sind:

aa) Vollstreckbarkeit

bb) Staatlicher Ursprung

cc) Bindende Natur

dd) Positive Festlegung

vgl. Hermann Kantorowicz „Der Begriff des Rechts“, Göttingen, o. J., S. 29. Wie Kantorowicz a. a. O selbst nachweist, sind sämtliche vier Merkmale, genau genommen, unbrauchbar und können deshalb auch hier im Text nicht als entscheidende Kriterien herangezogen werden.

b) Radbruch ("Vorschule der Rechtsphilosophie a. a. O., S. 34) liefert eine weitere Definition: Recht ist hiernach der "Inbegriff genereller, positiver Normen für das soziale Leben " (Normativ ${ }^{\alpha}$ heißt hier vor allem: wertend und fordernd. "Positiv ${ }^{\alpha}$ die Norm muß die „empirische "Gestalt eines Gesetzes oder einer Gewohnheit tragen, also positiv sein “ a. a. O. S. 34).

c) Nach soziologischer Betrachtungsweise sind für das Recht hauptsächlich drei konstitutive Elemente erforderlich:

a) eine räumlich, zeitlich und personell bestimmbare Kollektiveinheit als zwischenmenschliches Bezugssystem,

bb) die einzelnen, diesem Bezugssystem zugeordneten Menschen

cc) eine "letzten Endes bestimmende und maßgebende Instanz", welche Rechte und Pflichten zuweist und „irgendwie garantiert " (Wilhelm Bernsdorff: "Wörterbuch der Soziologie ${ }^{\star}$ Stuttgart 1969, 2. Aufl., Stichwort "Recht ${ }^{\alpha}$, S. 869).

Nach all diesen Kriterien wären nicht nur die Rechtsnormen aus den Jahren 1954/55, sondern auch die 122 Dokumente aus $1966 / 67$ als "Recht ${ }^{\alpha}$ anzusprechen. Selbst die strenge Anforderung der ${ }_{n} G a-$ rantie" wäre letzten Endes erfüllt.

Eine andere Frage ist, wie es um die Rechtsqualität bestellt ist, wenn der Normstifter gar kein Gesetz erlassen, sondern nur eine Empfehlung abgeben wollte. Ob hier die komplizierten juristischen Interpretationstechniken einzusetzen haben, die auf den mutmaßlichen Willen des "Gesetzgebers zurüdkgehen (vgl. dazu Heinrich Lehmann "Allg. Teil des BGB*, 8. Auflage, Bln. 1954, S. 53 f.) ist eine weitere Frage, die hier nicht im einzelnen beantwortet werden kann, da sie zu sehr vom Thema abführte. Im allgemeinen dürfte für die Ermittlung der Rechtssatzqualität wohl das äußere Erscheinungs bild der "Norm ${ }^{\alpha}$ maßgebend sein. (Zur „objektiven Theorie ${ }^{\alpha}$ vgl. Lehmann a. a. O. 54). Alles in allem also dürfte der im Text vertretenen Auffassung, daß es sich bei den hier zu behandelnden Bestimmungen um Anordnungen mit Rechtssatzqualität handelt, nichts im Wege stehen. 
zen waren ferner auf dem Höhepunkt der Kulturrevolution noch einigermaßen in der Lage, die Verwirklichung der von ihnen gesetzten Normen „irgendwie“ zu garantieren, und sei es auch nur durch den Einsatz solcher Organisationen, die ursprünglich nicht unbedingt für den Rechtsvollzug vorgesehen waren, wie Rotgardisten und Volksbefreiungsarmee ${ }^{\mathbf{3 6}}$.

\section{b) Auswertung der Tabelle II}

Ebenso verwirrend wie die Nomenklatur ist auch der Apparat, der die "Gesetze“ erläßt - wenigstens für die Periode 1966/67:

Zwölf verschiedene Organe und Organkombinationen übten zu dieser Zeit das Normsetzungsrecht aus, gar nicht zu reden von den Untereinheiten, die oft im zweiten Glied noch mitwirkten, wie z. B. das Allgemeine Amt des ZK, das Sekretariat des Staatsrates, das Amt für Auswärtige Angelegenheiten des Staatsrates, die Allgemeine Politische Abteilung der VBA usw.

Der Löwenanteil der "Gesetze“ stammt von vier Instanzen (ZK, ZK + ZKRA + StR, ZKMA, ZK + StR). Überragend ist die Stellung des ZK, das für sich allein $42 \%$ (= 51 Bestimmungen), in Verbindung mit dem Staatsrat 22\% und durch seine Ausschüsse (ZKMA, ZKKR) $18 \%$ aller „Rechtsnormen“ erlassen hat. Alles in allem ist es also mit nicht weniger als $81 \%$ (!) beteiligt. Die Leistung des Staatsrats nimmt sich daneben etwas kümmerlich aus: Von ihm allein stammen $5 \%$, aus seiner Zusammenarbeit mit anderen Organen $13 \%$; alles in allem also nur $18 \%$ ! Besonders nachdenklich sollte es stimmen, daß weder der Nationale Volkskongreß noch sein Ständiger Ausschuß zum Zuge gekommen ist.

Die Epoche 1954/55 bietet demgegenüber ein völlig anderes Bild. Es ist, als ob beide Systeme miteinander überhaupt nichts zu tun hätten. $77 \%$ der Legislative wurden dort vom Staatsrat und seinen Ministerien erledigt. Die Nationalversammlung war mit 10\%, der Ständige Ausschuß der Nationalversammlung mit $11 \%$ beteiligt. Nur 0,6\% fallen dagegen auf das ZK, der Rest auf die Staatsbank und den Staatspräsidenten.

Die Verhältnisse haben sich also grundlegend gewandelt. Beliefen sich die Anteile von Staatsrat und ZK in den Jahren 1954/55 auf das Verhältnis von 77:0,6, so lautete es zwölf Jahre später auf 18:82.

\section{c) Auswertung der Tabelle III}

Mit dem systematischen Abbau der Staatsorgane, insbesondere des Staatsrats, in dem sich ja die fachmännische Elite der Volksrepublik China konzentrierte ${ }^{37}$, wurden auch jene Kräfte desavouiert, die für eine exakte juristische Formulierung der Gesetze hätten sorgen können.

Kein Wunder also, daß die „Rechtsnormen“ der Epoche 1966/67 nicht mehr sauber durchdacht und nach Sachgebieten aufgegliedert sind. Da sie nur große Rahmen abzustecken und im übrigen die Spontaneität der Volksmassen auszulösen hatten, waren sie häufig so sehr mit Gefühlsmomenten angereichert, daß sich für

$36 \mathrm{Vgl}$. die zahlreichen Weisungen an Rotgardisten und an die Volksbefreiungsarmee in DB.

37 J. e. vgl. Donald Klein.,The State Council and the Cultural Revolution“, in China Quarterly (fortan CQ) Nr. 35 (Juli-Sept. 1968), S. $78 \mathrm{ff}$. 
eine klare Abgrenzung der "Gesetzgebungs"-Gegenstände kaum geeignete Kriterien finden lassen. Überdies befassen sich die meisten Bestimmungen mit der „Revolution im allgemeinen", wie folgende Beispiele beweisen: Soll etwa ein Befehl an die Rotgardisten, in ihre Schulen zurückzukehren, als Schulangelegenheit gewertet werden? Gehören Anorđ̄nungen an die VBA zum Schutz von Staatseigentum zu den „militärischen“ Regelungen? Ist der Befehl an die VBA, den Diebstahl von amtlichen Akten zu verhindern, eine Angelegenheit, die in den Bereich des „Militärs“ der „Rotgardisten“ oder des „inneren Dienstes“ gehört? Der Fragenkatalog ließe sich beliebig verlängern, soll aber in der hier gebotenen Kürze nur auf die Abgrenzungsschwierigkeiten hinweisen. Insofern kann die in Tabelle III wiedergegebene Einteilung nach "Gesetzesmaterien" nur den Wert einer groben Einschätzung beanspruchen, deren Unzulänglichkeit immerhin noch erträglicher erscheint als ein völliger Verzicht auf kategoriale Einstufung.

Wertvoll werden die hier gewonnenen Einsichten freilich erst, wenn man sie mit den Regelungen aus der Periode 1954/55 konfrontiert; denn dort herrschen in der Tat noch die klaren Regeln des juristischen Systembaus, bei dem auch auf klare Abgrenzung der "Gesetzesmaterien" geachtet wird. Lassen sich die damals ergangenen Bestimmungen noch eindeutig in einzelnen "Schubladen“ (Inneres, Außeres, Militär, Finanzen, Kultur, Landwirtschaft usw.) unterbringen, so bieten die Normen der Kulturrevolution einen "juristischen Brei“, in dem es kaum noch etwas zu sortieren gibt. Nicht zufällig finden sich deshalb unter der Rubrik „Allgemeines“ 81 Titel, während die Jahre 1954/55 an dieser Stelle nur 4 Normen beizusteuern haben.

Aus dieser Gegenüberstellung sollte deutlich werden, wie fadenscheinig die Rechtsnormen während der Kulturrevolution geworden sind. Der eigentliche Ausfüllungs- und Interpretationsprozeß wurde eben, wie nachfolgend noch zu erörtern ist, ganz nach unten verlagert, womit nicht zuletzt die zunehmende $E$ n $t s t$ a a $\mathrm{li} \mathrm{ch} \mathrm{u} \mathrm{g}$ des Entscheidungsprozesses deutlich wird.

d) Um wieviel „juristischer" die Regelungen der Periode I waren, vermag insbesondere auch ein Vergleich aus dem Bereich des Organisationsrechts zu verdeutlichen:

Die lokalen Regierungs- und Verwaltungsorgane wurden in der Periode I durch das ausführliche „Organisationsgesetz für die lokalen Volkskongresse und Volksräte" vom 21. 9. 1954 geregelt $^{38}$.

Die 42 Paragraphen dieses formellen (!) Gesetzes legen zunächst den genauen Standort für die lokalen Kongresse fest ${ }^{39}$. Nach einer Erörterung der Wahlmodalitäten $^{40}$ folgen genau definierte Kompetenzen ${ }^{41}$, sodann Regelungen über die Zusammensetzung ${ }^{42}$ und schließlich Bestimmungen über das Entscheidungsverfahren ${ }^{43}$. Indemnitätsvorschriften ${ }^{44}$ und Regelungen über das Verhältnis der Abgeordneten zu ihrem Wahlkreis schließen sich $a^{45}$. Die Bestimmungen über die lokalen Volksräte ( $\left(\$ \int 23-42\right)$ sind analog geregelt.

$38 \mathrm{Vgl}$. die Sammlung ${ }_{n} \mathrm{fa}-\mathrm{kui}{ }^{\star}$ (FN. 27) Bd. II, S. 139-150.

39 S $1,2.2$

41 S 6-7 (24 Ziffern!)

42 is $8-12,15,16$

43 of $13,14,17$

44 \ड 18,19

45 is $20,21$. 
Selbst aus der Sicht westlicher Jurisprudenz nimmt sich dieses Gesetz gut aus. Die Gliederung ist sauber, die Systematik gelungen und das Detail so ausführlich, daß kaum Lücken vorhanden sein dürften.

Eine ganz andere juristische Landschaft eröffnet sich, wenn man das Augenmerk nun auf die entsprechende Regelung während der Kulturrevolution richtet: Jetzt sind es die Revolutionskomitees, die an die Stelle der ehemaligen lokalen Kongresse und Ausschüsse gerückt sind. Obwohl diese neuen revolutionären Organe schon im Januar 1967 "aus den Stürmen der Kulturrevolution heraus geboren“ wurden, existiert für sie bis heute noch keine Organisationsnorm, die sich im juristischen Sinne als solche bezeichnen dürfte. Dies ist um so bemerkenswerter, als die Revolutionskomitees nicht nur auf den engen Bereich der lokalen Organe beschränkt sind, sondern weit darüber hinaus praktisch jeder wirtschaftlichen pädagogischen, administrativen und kulturellen "Einheit“ eine neue organisatorische Form aufgeprägt haben ${ }^{46}$.

Die juristische Durchdringung der Revolutionskomitees ist denkbar unvollkommen. In dem „16-Punkte-Beschluß“ vom 8. August 1966 waren sie nur andeutungsweise enthalten ${ }^{47}$. Erst das Beispiel der Provinz Shanhsi, wo am 12.1.1967 eine „Dreier-Allianz“48 den Parteiausschuß der Provinz entmachtet und dann selbst die Herrschaft an sich gerissen hatte, wirkte als auslösendes Moment, nicht zuletzt deshalb, weil diese Dreier-Allianz ihre „revolutionären Aufgaben“ wesentlich effektiver zu lösen vermochte als die Aktionsgemeinschaft der ohne militärische Unterstützung arbeitenden „revolutionären Rebellen“, die ihr Glück gleichzeitig in Shanghai versucht hatten. Nicht zuletzt dieser Erfolg veranlaßte die Pekinger Führung bereits am 23.1.1967 zu einem Tagesbefehl, der den Soldaten der Volksbefreiungsarmee nahelegte, ihre bisher passive Haltung aufzugeben und nunmehr die "Linke“ zu unterstützen". Auch die amtliche Presse wurde immer mehr auf das Shanhsi-Modell aufmerksam. Bereits am 31. Januar 1967 konstituierte sich das Provinzrevolutionskomitee von Heilungchiang, und etwas später gab Mao seine bekannte „Direktive über die Machtergreifung durch DreierAllianzen" heraus ${ }^{50}$.

In dieser Aufbruchstimmung wurden zwar auch einige unbeholfene juristische Formulierungen „über die Errichtung vorläufiger Machtorgane" versucht ${ }^{51}$; im allgemeinen unterblieb jedoch jegliche Fixierung. Das revolutionäre Geschehen allein sollte die auf Dreier-Allianzen aufgebauten "Revolutionskomitees" formen. Vor allem die militärischen Abteilungen, die in jedes Revolutionskomitee nach dem Grundsatz der Drittelparität eingebaut wurden, sorgten dafür, daß die einzelnen Ausschüsse in der Tat allmählich ein einheitliches Gepräge bekamen.

46 Vom unterschiedlichen Geist beider "Gesetzgebungsperioden zeugen im übrigen auch die beiden Parteisatzungen von 1956 (8. Parteitag) und 1969 (9. Parteitag), obwohl beide Regelungen jeweils erst in einer Zeit erlassen wurden, da der Umschwung der Gesetzgebungspolitik bereits wieder eingesetzt hatte.

46 Umfaßt das Statut von 19569 Kapitel, 60 Paragraphen, 15000 Schriftzeichen und eine eigene ausführliche Präambel, so ist die Satzung von 1969 auf 6 Kapitel, 12 Paragraphen und rd. 2700 Zeichen zusammengeschrumpft - ganz zu schweigen von der Präambel, die gleich mit in $\$ 1$ eingebaut wurde. Dabei ist hier nur vom äußeren Erscheinungsbild die Rede. Der Inhalt selbst ergibt noch krassere Unterschiede. (Im einzelnen vgl. dazu „Issues and Studies ${ }^{\alpha}$ Vol. V Nr. 6, March 1969, S. 15 ff., CNA Nr. 757).

47 9, vgl. Peking Rundschau $1966 \mathrm{Nr}$. 33, S. $6 \mathrm{f}$.

485 ,führende Kader “, die früher selbst der Regierung bzw. dem Provinzparteiausschuß von Shanhsi angehört hatten; einige Repräsentanten sog. "revolutionärer Rebellenorganisationen“ und Vertreter einiger VBA-Einheiten.

49 Beschluß des ZK, des ZKMA u. des ZKRA, die „feste Unterstützung der revolutionären Linken durch die VBA ${ }^{\alpha}$ betreffend, vom 23. 1. 1967 (DB, S. 195 ff.).

50 Rote Fahne (fortan RF) 1967, Nr. 4, S. 20.

51 Vgl. etwa den entsprechenden Beschluß des Vorbereitungskomitees der Provinz Chianghsi, in ${ }_{n}$ Facts and Features*, vol. I. Nr. 4 v. 13. 12. 1967, S. 9 ff., und die Stellungnahme dazu bei O. Weggel "Die chinesischen Revolutionskomitees“ (Mitteilungen des Instituts für Asienkunde, H. H.), Hamburg 1968, S. 69. 
Nichts kann die Metamorphose der chinesischen "Gesetzgebung“ während der letzten Jahre deutlicher veranschaulichen als der Vergleich zwischen diesen beiden organisationsrechtlichen Regelungen. War das Gesetz von 1954/55 noch ganz von institutionellem Denken beherrscht, von Experten erarbeitet und nach soliden handwerklichen Methoden aufgebaut, so blieb die Gestaltung der kulturrevolutionären Führungsapparatur ganz der „Schöpferkraft der Massen“ überlassen, zumindest der Theorie nach (in Wirklichkeit sprach ja die Volksbefreiungsarmee, die mit den Massen durchaus nicht identisch ist, in jeder Einheit ein ziemlich entscheidendes Wort). Die Zentrale fungierte nur als eine Art Transmissionsstation, die die Fülle der "revolutionären Erfahrungen" sichtete, sie zusammenfaßte und wieder an die „Massen“ zurückfütterte ${ }^{52}$.

Wenn etwa Mao Tse-tung gleich nach dem Auftauchen der ersten Dreier-Allianzen die „Weisung“ erließ, daß „Revolutionskomitees gut"52a seien, so war dies eine "Direktive“, die an Einfachheit und Kürze schlechthin nicht mehr zu unterbieten ist.

Der juristische Sachverstand weigert sich, hier überhaupt noch in den Kategorien von Recht und Rechtsnormen zu denken. In der Tat stößt man an dieser Stelle in Bereiche vor, die vielleicht nicht mehr juristisch, sondern nur noch soziologisch zu bewältigen sind und die sich mit Max Webers Kategorien der „legalen“ und der "charismatischen" Herrschaft noch am besten veranschaulichen lassen: Es war kein Zufall, daß Maos „Vergöttlichung“ gerade in dem Augenblick besonders nachhaltig einzusetzen begann, als unter den ersten Stürmen der Kulturrevolution die etablierte Autoritätskette zu zerreißen begann. Je mehr die „legale“, auf Gesetze und Institutionen gegründete Herrschaft der Partei und des Staatsapparats dahinschmolz, um so herrischer begann sich der Anspruch Maos und seiner Quadriga ${ }^{53}$ auf (affektuelle) Hingabe an ihren „charismatischen“ Willen zu entfalten.

Gerade zu dieser Zeit konnte man versucht sein, die Direktiven, die ab und zu von diesem "magischen" Zentrum ausgingen, mit einem Signal zu vergleichen, das von Zeit zu Zeit aufblitzt und dann jedesmal den Pawlow'schen Effekt fieberhafter Aktivität in den einzelnen Einheiten auslöste. Was „von oben“ kam, war im allgemeinen keine praktische Handlungsanweisung mehr, die von einer routinierten Verwaltungsmaschinerie zurechtgefiltert wurde, sondern häufig nur noch eine Befehlshülse, welche eigentlich nur noch den einen Zweck hatte, die Spontaneität der Massen wachzurufen oder wachzuhalten. Was konkret zu tun war, blieb den jeweiligen Einheiten überlassen, letzten Endes also meist den Revolutionskomitees. Es kann kaum bezweifelt werden, daß die einzelnen „Einheiten“ mit diesem überdimensionalen „Gesetzes“-Rahmen ${ }^{54}$, der durch den Wegfall der routinierten Bürokratie nur noch vergrößert wurde, zumeist überfordert waren.

52 Mit dieser Beschreibung sind bereits die wesentlichen Merkmale der „Massenlinie ${ }^{\alpha}$ wiedergegeben: Die Massenlinie ist die grundlegende Arbeitsmethode durch welche die komm. Kader versuchen, zwischen sich und der chinesischen Bevölkerung eine einheitliche Beziehung herzustellen und auszubauen - und damit die Unterstützung und aktive Teilnahme des Volkes zu erlangen ${ }^{\alpha}$. Diese Methode umfaßt $\mathbf{z w e i}$ Techniken: „von den Massen zu den Massen ${ }^{\star}$ und „Verbindung des Allgemeinen mit dem Besonderen ${ }^{\star}$. Vgl. John Wilson Lewis, „Leadership im Comm. China“. Ithaca/NY 1963, S. 70, vgl. auch die umfassende Definition der Massenlinie in Abs. 9 der Präambel zur Parteisatzung von 1956.

52a RF $1968 \mathrm{Nr}, 1, \mathrm{~S}, 17 \mathrm{ff}$

$53 \mathrm{ZK}, \mathrm{ZKMA}, \mathrm{ZKRA}$, StR, vgl. Tabelle II.

54 Es wäre etwas handlicher gewesen, hier den Begriff „Ermessensbereich ${ }^{\alpha}$ zu verwenden. Nichts wäre freilich falscher als diesen, in der deutschen Verwaltungswissenschaft so prominenten Begriff in eine Erörterung über chinesisches Recht einzubringen. Die herrschende Lehre in Deutschland versteht darunter nämlich einen Spielraum des Handelns und Entschlusses, der die Wahl zwischen mehreren, in gleicher Weise möglichen Arten des Sich-Verhaltens zuläßt (Ernst Forsthoff "Lehrbuch des Verwaltungsrechts ${ }^{\alpha}$, 5. Aufl., München und Berlin 1955, S. 68). Ermessen ist somit "wählendes Verhalten im Rahmen einer 5. Aufl., München und Berlin 1955, S. 68). Ermessen ist somit wwählendes Verhalten im Rahmen einer
Wertverwirklichung ${ }^{\alpha}$ (ibd. S. 71). Von einer solchen Wahlf reiheit kann im China Maos keine Rede sein: Das gesamte Denken hat dort durch das Nadelöhr der Dialektik zu gehen, die den sozialen Bezugs- 
So erklärte sich denn auch die hektische Betriebsamtkeit, mit der Revolutionskomitees aller Schattierungen unter "Mithilfe“ der Volksbefreiungsarmee den jeweilig „neuesten Weisungen" - koste es, was es wolle - konkrete Handlungsorientierungen $\mathrm{zu}$ entlocken versuchten ${ }^{55}$. Niemals waren andererseits die (theoretischen!) Chancen zur „Selbsterziehung der Massen“ größer als während der kurzen Zeitspanne 1966/67.

Seitdem ist die Volksrepublik längst wieder zu einer gewissen „Normalität“ zurückgekehrt. Die Ausnahmesituation am Beginn der Kulturrevolution war hier auch nur deshalb so ausführlich zu erörtern, weil sie in besonders paradigmatischer Weise das „Gesetzgebungsverfahren“ in einer Gesellschaft verdeutlicht, die — von revolutionärem Eifer besessen - allen institutionellen Ballast über Bord geworfen hat und ganz der Weisheit der Volksmassen das Steuer überläßt ${ }^{56}$. Idealtypisch gesehen stünde am Ende einer solchen Entwicklung eine Gemeinschaft von Befehlsempfängern, die sich ihre „Befehle“ selbst gibt. Die "Gesetze" würden sich in einer solchen Gemeinschaft durch den bloßen Antrieb innerer Notwendigkeit herausbilden und innerhalb einer "juristischen Sekunde“ bereits in Realität verwandeln. Das soziale Modell Talcott Parsons, der die Identifizierung von objektiven Gemeinschaftsnormen und individuellen Motivierungen (values motives and systems of action) zum Ideal einer integrierten Gesellschaft erklärt ${ }^{57}$, wäre damit Wirklichkeit geworden.

Ein institutioneller Gesetzgeber, der Normen definiert und ihre Verwirklichung garantieren müßte, wäre in einem solchen sozialen Rahmen überflüssig. Das ist ein Wunschtraum, aber zugleich auch ein ziemlich wirksames utopisches Element in Maos Denken, das auf die chinesische Politik von Zeit zu Zeit erheblichen Einfluß ausübt und beispielsweise dazu führt, die permanente Revolution in Form immer neuer „Kulturrevolutionen“ zu proklamieren. Da Mao den Glauben, daß menschliche Unzulänglichkeit immer dieselbe bleibe und daß soziale Experimente deshalb von vornherein zum Scheitern verurteilt seien, als „Metaphysik“ verwirft ${ }^{58}$, ist das oben aufgezeigte Modell einer Gesellschaft ohne institutionellen Gesetzgeber als ein Ideal anzusehen, das wohl auch in den nächsten Jahren immer wieder angepeilt wird.

Ob die Maoisten mit ihrem Glauben recht behalten können, wird die Geschichte zeigen. Einstweilen freilich klafft zwischen Ideal und Wirklichkeit noch ein tiefer Abgrund, und deshalb bleibt der Pekinger Führung nichts anderes übrig als mit der Realität immer neue Kompromisse zu schließen. Sie wird also, konkret gesprochen, ohne "Gesetzgebung" nicht auskommen, wie immer sie solche "Gesetze" auch kaschieren mag. Nur eine durch Selbsterziehung vollkommen geläuterte „Volksmasse“ könnte den chinesischen Traum von einer ganz dem Pneuma hingegebenen, ohne Institutionen auskommenden Gesellschaft Wirklichkeit werden lassen. Man sieht: Das Wunschbild einer gesetzesfreien Gesellschaft hängt aufs engste mit dem Traum vom „Neuen Menschen“ zusammen.

rahmen in ein Raster von Widersprüchen auflöst. Jede Entscheidung läßt deshalb nur zwei Möglich* keiten zu: entweder dafür oder dagegen. Eine mittlere Lösung entfällt somit und disqualifiziert denjenigen, der sich für ein $Z$ wischendrin entscheiden möchte, als Abweichler. - Richtiger wäre es deshalb schon, die Rahmenausfüllung als Interpretation eines, unbestimmten Rechtsbegriffs zu begreifen, wobei das "Denken Mao Tse-tungs ${ }^{\alpha}$ die Auslegungsmaßstäbe abgäbe. Viel ist mit einer solchen Subsumtion freilich nicht gewonnen!

55 Weggel, Revolutionskomitees a. a. O., S. 91.

56 Näheres dazu vgl. unten B. II 1 .

57 Talcott Parsons, "The Social System ${ }^{\star}$ New York, London, 3. Auflage 1966, S. $207 \mathrm{ff} ., 36 \mathrm{ff}$.

58 . Ober den Widerspruch ${ }^{\alpha}$, Mao Tse-tung, „Ausgewählte Werke ${ }^{\alpha}$ (foran MTt AW), Bd. I, Peking 1968, S. $366 \mathrm{ff}$. 
e) Die Darstellung der rechtlichen Erscheinungsformen im kommunistischen China bliebe unvollkommen, würden hier nicht einige der merkwürdigsten „Rechtsquellen " erwähnt, die die Kulturrevolution hervorgebracht hat.

Es war oben von der "charismatischen Herrschaft" Mao Tse-tungs einerseits und von der damit korrespondierenden "schöpferischen Spontaneität der Volksmassen“" andererseits die Rede. $\mathrm{Zu}$ welch eigenartigen Auswirkungen eine solche Konzeption führen kann, zeigen besonders deutlich die sogenannten „Neuesten Weisungen des Vorsitzenden Mao", die seit der Reise des chinesischen Parteiführers durch einige der Schlüsselprovinzen Nordchinas im September 1967 plötzlich in der Presse auftauchten und seitdem zur Regel geworden sind.

In diesen beinahe an Aphorismen gemahnenden wortkargen Befehlsfragmenten werden Grundlinien der Politik bestimmt, und es ist kein Zufall, daß sie stets dann auftauchen, wenn die Versuchsperiode für einen bestimmten politischen Teilbereich abgeschlossen ist.

Presse, Radio und der sonstige Apparat der Massenkommunikationsmittel scheuen keine Mühe, jede dieser "Weisungen" mit einem Maximum an Propagandaaufwand zu verbreiten. Nicht zuletzt deshalb gehören Maos Direktiven heute zu den weitaus bekanntesten "Rechtsnormen“ in der Volksrepublik China. Es mag vorkommen, daß die „Massen“ mit den offiziellen Gesetzen und Verordnungen nicht immer auf gutem Fuße stehen; es ist aber schlechterdings unmöglich, die „Weisungen des Vorsitzenden" nicht zu kennen!

In Grundsatzartikeln wird immer wieder betont, daß diese Neuesten Weisungen „mit Eifer studiert, in beispielhafter Weise angewandt, mit Begeisterung verbreitet und entschlossen verteidigt werden müssen ... Die Einhaltung dieser Direktiven ist nicht nur eine Frage der Arbeitsmethode, sondern ein Element der politischen Richtung bei der Unterstützung der Linken ${ }^{59}$ “.

Die Weisungen tragen im allgemeinen kein Datum und können deshalb nur immer nach dem Tag ihrer Veröffentlichung identifiziert werden. Nachfolgend seien einige „Neueste Weisungen des Vorsitzenden Mao“ in der Reihenfolge ihres Erscheinens aufgeführt.

1. Zum Thema „Arbeiterklasse“: „Es gibt keinen grundsätzlichen Widerspruch zwischen den Interessen der Arbeiterklasse. Solange das Proletariat seine Diktatur ausübt, gibt es keinerlei Grund für die Arbeiterklasse, sich in zwei große unversöhnliche Organisationen aufzuspalten ${ }^{60}$."

2. Zum Thema „Rotgardisten“: „Die revolutionären Rotgardisten und revolutionären studentischen Massenorganisationen sollen die revolutionäre Große Allianz verwirklichen. Revolutionäre Massenorganisationen sollen ebenfalls die Große Allianz nach revolutionären Prinzipien schaffen ${ }^{61}$."

3. Zum Thema „Egoismus“: „Es ist unbedingt notwendig, selbstische Interessen zu bekämpfen und den Revisionismus zu kritisieren ${ }^{62 . “}$

4. Zum Thema „Erziehung“: „Die proletarische Erziehungsrevolution soll sich auf die Massen der revolutionären Studenten, Lehrer und Arbeiter stützen, indem man vor allem den aktivsten unter ihnen das Mandat anvertraut, vor allem also jenen proletarischen Revolutionären, die entschlossen sind, die Große Proletarische Kulturrevolution bis zum Ende zu führen"3."

59 NCNA 1. I. $68 /$ S. 4

60 RF 1967, Nr. 15, S. 2.

$61 \mathrm{RF} 1967, \mathrm{Nr} .15, \mathrm{~S} .3$.

62 VZ 6. Okt. 67.

$63 \mathrm{VZ}$. Nov. 69 . 
5. Zum Thema „Partei“: „Die Partei soll sich aus fortgeschrittenen Elementen des Proletariats zusammensetzen; sie soll eine lebendige Organisation von Vorkämpfern sein, welche das Proletariat und die revolutionären Massen im Kampfe gegen den Feind zu führen vermag64."

6. Zum Thema "Studium“: „Studienklassen einzurichten ist eine hervorragende Methode. Durch solche Studienkurse lassen sich eine ganze Menge von Problemen lösen ${ }^{65}$."

7. Zum Thema „Kader“: „Den Kadern soll vor allem und an erster Stelle durch Erziehung geholfen werden ${ }^{66}$."

8. Zum Thema „Revolutionskomitees“: „Die Grunderfahrung der Revolutionskomitees läßt sich folgendermaßen zusammenfassen: Sie sind dreiteilig und setzen sich aus Vertretern der revolutionären Kader, Vertretern der Streitkräfte und Repräsentanten der revolutionären Massen zusammen. Auf diese Weise ergibt sich eine revolutionäre Dreier-Allianz. Das Revolutionskomitee soll sich um einheitliche Führung bemühen, überflüssige oder sich überschneidende Verwaltungsstrukturen abschaffen, das Prinzip „Bessere Truppen und einfache Verwaltung“ befolgen und eine revolutionierte Führungsgruppe einsetzen, die mit den Massen engen Kontakt hältt ${ }^{67}$.“

9. Zum Thema „Kulturrevolution“: „Die Große Proletarische Kulturrevolution ist letztlich eine große politische Revolution unter den Bedingungen des Sozialismus, welche vom Proletariat gegen die Bourgeoisie und alle anderen Ausbeuterklassen geführt wird; sie ist eine Fortsetzung des verlängerten Kampfes zwischen der KPC und den revolutionären Massen unter ihrer Führung einerseits und den Kuomintang-Reaktionären andererseits - eine Verlängerung also des Klassenkampfes zwischen dem Proletariat und der Bourgeoisie ${ }^{68}$."

10. Zum Thema „Erziehung“: „Auch jetzt brauchen wir noch Hochschulen. Ich möchte betonen, daß wir naturwissenschaftliche und technische Hochschulen unterhalten sollen; freilich muß die Schulungsperiode abgekürzt, die Erziehung revolutioniert, proletarische Politik an die erste Stelle gesetzt und jenes Beispiel befolgt werden, das von den Arbeitern der Werkzeugmaschinenfabrik Shanghai gesetzt wurde. Studenten müssen also aus der Reihe der Arbeiter und Bauern, die ja über praktische Erfahrung verfügen, ausgewählt werden und haben nach ihrem Studium wieder in die praktische Produktion zurückzukehren ${ }^{69}$."

11. Zum Thema "Journalismus": "Zeitungen dürfen nicht hinter verschlossenen Türen angefertigt werden. Vielmehr sollen sie immer die Massen vor sich haben, und gleichzeitig muß ihre allgemeine Orientierung frisch und lebendig sein ${ }^{70}$."

12. Zum Thema "geschichtliche Erfahrung“: „Historische Erfahrung soll unsere Aufmerksamheit in Anspruch nehmen. Die Linie und der Standpunkt müssen immer und immer wieder überprüft werden. Man soll aber nicht nur mit ein paar wenigen Leuten darüber sprechen, sondern sie den revolutionären Massen bekanntmachen ${ }^{71}$."

13. Zum Thema „hsia fang“: „Es ist notwendig, daß junge Leute mit höherer Schulbildung aufs Land gehen und von den armen Bauern und unteren Mittel-

\footnotetext{
64 VZ 1. I. 1968 + RF 1968, Nr. 1, S. 1.

$65 \mathrm{VZ}$ 3. II. 1968.

66 NCNA 17. II. $1968 / 3$.

67 VZ 30. III. 1968

68 VZ 15. IV. 1968.

$69 \mathrm{VZ} 22$. VII. 1968.

70 RF 1968, Nr. 2, S. 9.

71 VZ 25. XI. 1968.
} 
bauern rückerzogen werden. Kader und andere Städter sollten überredet werden, ihre Söhne und Töchter, welche die höhere Schule oder die Universität beendet haben, aufs Land zu schicken. Wir wollen alles mobilisieren. Die Genossen im ganzen Lande werden sie willkommen heißen ${ }^{72 . " ~}$

Es war notwendig, hier eine einigermaßen repräsentative Auswahl der „Neuesten Weisungen" zu geben; andernfalls müßte dieses Element unverständlich bleiben. Selbstverständlich ließe sich die Reihe fortsetzen; doch wurde hier darauf geachtet, eine Reihe von Richtlinien anzuführen, die besonders fundamentale Fragen betreffen. Es kann kaum ein Zweifel bestehen, daß diesen Weisungen Maos unmittelbar "rechtliche" Verbindlichkeit zukommt. Vor allem der Elan und die Akribie, mit denen sie jeweils publik gemacht werden, zeigt an, daß die "Weisungen“ keinen Widerspruch dulden, sondern bedingungslosen Vollzug gebieten. Von der juristischen Effizienz her gesehen, nehmen sie unter allen „Rechtsquellen“ sogar den höchsten Rang ein, so daß allen anderen Normen gegenüber das Prinzip des „lex superior derogat legi posteriori“ zur Anwendung kommt.

Diese unmittelbare Geltung verhindert freilich nicht, daß die Weisungen Mao Tsetungs manchmal noch zum Gegenstand einer besonderen Rechtsnorm gemacht werden. Dies ist z. B. besonders häufig der Fall bei der berühmten Direktive vom 7. März 196773. Zu einem Teil freilich erklärt sich hier der Erlaß eines besonderen Gesetzes dadurch, daß diese "Weisung“ nicht direkt an die Öffentlichkeit, sondern an die "Genossen Lin Piao, Chou En-lai und an die Genossen in der Kulturrevolutionsgruppe" gerichtet war ${ }^{74}$. Aber auch solche Weisungen, die unmittelbar an die Massen adressiert sind, werden zum Teil - nach dem Grundsatz „Doppelt genäht hält besser “ - nochmals zum Gegenstand eines Gesetzes gemacht ${ }^{75}$.

Oben wurde bereits erwähnt, daß viele "Gesetze“ nur den Zweck haben, die Spontaneität der Massen wachzurufen ${ }^{76}$. Für „die letzten Weisungen des Vorsitzenden Mao“ gilt diese Erkenntnis in ganz besonderem Maße.

\section{Deutung.}

In Abschnitt B I sollte eine Bestandsaufnahme der chinesischen "Gesetzgebung“ versucht werden. Es gilt nun, die verschiedenen disparaten Elemente zu deuten und ihnen einen systematischen Standort in der Ideologie und Gesellschaftsstruktur der Volksrepublik China anzuweisen.

Hierbei ist das Recht vor allem mit drei wichtigen Phänomenen zu konfrontieren, nämlich mit der „Massenlinie“, dem Praxisdogma und schließlich mit der Theorie der Widersprüche.

\footnotetext{
72 VZ 22. XII. 1968.

73 DB S. 356: Mao legte hier seine Vorstellung über die soziale Erneuerung nieder (Formel: Arbeiter Bauer - Soldat - Intellektueller usw. J e d e r soll a 11 e Funktionen ausfüllen!)

74 DB S. 566.

75 DB S. $368,345$.

76 Oben B. I, 2. d.
} 


\section{Das Recht und die „Massenlinie“}

Eines der Leitmotive sino-kommunistischen Denkens, die Massenlinie, hat auch im Bereich der Gesetzgebung Eingang gefunden, besonders seit dem VIII. Parteitag im Jahre 1956. Gerade unter ihrer Einwirkung wurde das gesamte Feld der Gesetzgebung so stark umgepflügt, daß der legislative Bereich als solcher fast nicht mehr identifizierbar war.

Vielleicht läßt sich die Metamorphose, die hier innerhalb weniger Jahre vor sich gegangen ist, am besten an Hand der Vorgänge im administrativen System illustrieren. Die Verhältnisse in diesem Bereich sind schon so gut erforscht, daß sich der Umweg lohnt, zumal ja die verschiedenen staatlichen Gewalten, die ursprünglich noch relativ auseinandergehalten wurden, im Laufe der Zeit immer mehr miteinander verschmolzen77, so daß "Gesetzgebung " und „Verwaltung“ schließlich fast zur Deckung kamen ${ }^{78}$.

Als die Kommunisten 1949 im Zeichen der "Neuen Demokratie“78 den Verwaltungsapparat der Kuomintang übernahmen, erbten sie ein System, das vor allem durch zwei Besonderheiten geprägt war:

a) Horizontal waren die Verwaltungsorgane „ressortsystemorientiert“, wofür die hochspezialisierten Maschinenbauministerien ein gutes Beispiel abgeben. b) Diese Verfachlichung herrschte auch im vertika le n Bereich. Jedes Ressort hatte seinen eigenen, streng auf Kompetenzen eingeschworenen Verwaltungsunterbau, der wie ein Strang von der Zentrale bis zur untersten Instanz durchlief und in dem die Befehle reibungslos nach unten und die Informationen nach oben fließen konnten. Die Verwaltungsfunktionen waren also mit anderen Worten "horizontal dekonzentriert" und „vertikal konzentriert"79.

Solange die Herrschaft hochqualifizierter Fachleute unentbehrlich war (Zeit des ersten Fünfjahresplans! $)^{80}$, schienen das Ressortprinzip und eine gewisse vertikale Konzentration unverzichtbar.

Die große Wende setzte mit dem VIII. Parteitag (1956), besonders aber mit der „Dreibanner-Politik“ (1958) ein, bei der die Mass e n endgültig jenes Mandat übernahmen, das ihnen nach den theoretischen Äußerungen Maos längst zukam. Nun wurde auch das Verwaltungssystem (maoistisch gesehen) endgültig vom Kopf auf die Beine gestellt: An die Stelle des Ressortsystems trat der "A u s s h u t y p " mit seinen fachlich undifferenzierten, mehrere Ressorts pau-

$77 \mathrm{Vgl}$. Verfassung a) Abschn. 6 (Art. 73 ff.): richterliche Gewalt; b) Abschn. 3 (Art. 47!): Verwaltung; c) Abschn. 1 (insbes. 22, 27): Gesetzgebung.

78 Vgl. dazu MTt, AW Bd. II, Peking 1968, S. $395 \mathrm{ff}$

$79 \mathrm{Im}$ Sinne der deutschen Verwaltungsrechtswissenschaft werden hier die Begriffe „Konzentration* und "Dekonzentration ${ }^{\alpha}$ sowie das Begriffspaar „Zentralisation ${ }^{\alpha}$ und „Dezentralisation ${ }^{*}$ verwendet.

Die Begriffe "Konzentration " und "Dekonzentration“ beziehen sich nur auf Gliederungsprinzipien innerhalb der. Verwaltung, in deren Bereich sie vor allem in zwei Richtungen wirksam werden: Die Konzentration in horizon tale r Richtung besteht in dem Bestreben, nach Möglichkeit alle Funktionen einer Verwaltungsstufe in e i e r Behörde zu vereinen, während bei Dekonzentration meh $r e$ Behörden eingeschaltet werden.

Die vertika le Konzentration oder Dekonzentration hat mit der Verteilung der Funktionen zwischen den unteren Instanzen und der Verwaltungsspitze zu tun: Soweit das Schwergewicht der Verwaltungstätigkeit in die Verwaltungsspitze verlagert wird, spricht man von vertikaler Konzentration. Werden die Aufgaben jedoch möglichst auf die Mittel- und Unterbehörden verteilt, so liegt vertikale Dekonzentration vor.

"Zentralisation“ und "Dezentralisation“ betreffen demgegenüber ein ganz neues Beziehungsfeld:

Von "Zentralisation" spricht man, wenn die Verwaltungsaufgaben bei der staatlichen Verwaltung zusammengefaßt werden. Von „Dezentralisation“ ist andererseits die Rede, wenn die Verwaltungsaufgaben von der staatlichen Verwaltung an selbständige Verwaltungseinheiten zu selbständiger Erledigung übertragen werden.

Zum Ganzen vgl. Ernst Forsthoff, „Lehrbuch des Verwaltungsrechts“, 1. Bd., Algemeiner Teil. München und Berlin 1955, S. $354 \mathrm{f}$.

80 Der Plan (1953-57) stand ganz unter dem Einfluß des sowjetischen Vorbildes und machte deshalb Spezialisten erforderlich. 
schal zusammenfassenden Funktionen ${ }^{81}$. Die Partei vor allem war die ideale Pflanzstätte für diese neue Form des Administrierens. In ihr saßen nicht „Beamte“, sondern „Kader", für die ein ganz anderes Arbeitsethos gilt als für den Typ des Bürokraten: nicht Arbeitsteilung, sondern Allzuständigkeit ${ }^{82}$, nicht Spezifizierung von Rollen und Funktionen, sondern ihre Verwischung, und nicht Aufteilung zwischen Befehlserteilung und -ausführung, sondern deren Zusammenfassung una persona wurden zum neuen Ideal. Auch jenes laisser faire, das bisher den unpolitischen Experten frei hatte schalten lassen, mußte nun dem Grundsatz weichen, daß politische Gesinnung wichtiger sei als berufliche Tauglichkeit („rot vor fachmännisch“). Nicht der routinierte Bürokrat war nunmehr gefragt, sondern die mitreißende Führerpersönlichkeit, die durch ihr Beispiel zu überzeugen und dadurch die Massen für die Ziele der Führung $\mathrm{zu}$ begeistern verstand.

Die Partei setzte sich aber nicht nur über das im horizontalen Bereich etablierte Ressortprinzip hinweg, sondern schaltete sich auch in die Befehlsvertikale ein mit der Folge, daß die Verwaltungsorgane von nun an auf zwei „Herren“ zu horchen hatten. Diese "Doppelherrschaft" 83 wirkte sich im Sinne einer vertikalen Dekonzentration aus. Mit dem Erstarken der Partei setzte sich also das „Ausschußprinzip" durch, das sein Echo in horizontaler Konzentration und vertikaler Dekonzentration fand und damit die frühere Organisationsstruktur in ihr Gegenteil verkehrte.

Die Partei blieb diesen neuen Organisationsprinzipien zehn Jahre lang treu. Mit dem Einsetzen der Großen Proletarischen Kulturrevolution im Jahre 1966 freilich veränderte sich die Situation wiederum schlagartig: Sowohl der Verwaltungs- als auch der Parteiapparat ging vor allem auf den unteren Rängen fast völlig in die Brüche. Auf dieser Trümmerlandschaft entstanden - nach den mißlungenen Experimenten mit der "Pariser Kommune" - seit Ende Januar 1967 neue „revolutionäre Führungsorgane“, die Revolutionskomitees, die „mit proletarischer Autorität" die Aufgaben von Partei und Staat in den einzelnen administrativen, erzieherischen, industriellen, landwirtschaftlichen und kulturellen „Einheiten" übernahmen"

Jedes dieser Revolutionskomitees folgte dem System der Drittelparität und war dementsprechend aus Kadern, Soldaten und den Vertretern von Massenorganisationen zusammengesetzt. Damit war die Macht endgültig ganz den $\mathrm{M}$ as se $\mathbf{n}$ zugefallen ${ }^{85}$.

Aus dieser historischen Entwicklung ließe sich eine idealtypische Verschiebung von der Staatsführung über die Parteiführung zur Massenführung und somit eine Versöhnung der Wirklichkeit mit der Idee der Mas sen lin i e feststellen. Das Prinzip des Ausschußsystems und der vertikalen Dekonzentration wurde mit der Machtergreifung der Revolutionskomitees indes selbstverständlich nicht abgeschafft, sondern eher bekräftigt.

81 Vgl. Franz Schurmann „Ideology and Organization in Communist China ${ }^{\alpha}$, Second Edit., Berkeley and Los Angeles 1968, S. 178, spricht von "committee-type-agency“.

82 to-mien-shou ( $n$ vielseitige Hände“ $!$ ).

83 Schurmann a. a. O., S. 189, spricht von „dual rule“.

84 Vgl. Weggel a. a. O., S. 75.

$85 \mathrm{Da}$ die Volksbefreiungsarmee immer stärkere Macht in den Revolutionskomitees ausübt, erhebt sich allerdings die Frage, ob wirklich die "Massen“ herrschen. Diese Frage wirft das weitere Problem auf, ob auch die Soldaten in Wirklichkeit (nicht nur der Definition nach!) zu den "Massen“ gehören, oder ob ihr militärischer Status mit seinem spezifischen Lebensstil nicht etwas „Besonderes ${ }^{\alpha}$ aus ihnen gemacht hat, das sie von den Massen abhebt. - Für die vorliegende Darstellung, die ja auf die gesetzesausführende Tätigkeit der einzelnen Einheiten abstellen will, ist diese Frage freilich letzten Endes unerheblich und kann hier unbeantwortet bleiben. 
Mit dem Wiederaufbau der Partei seit April 1969 entsteht freilich eine ganz neue Situation; denn Partei und Revolutionskomitee stehen zueinander „im Verhältnis des Führers zum Geführten "86.

Bisher war immer nur vom administrativen System die Rede. Doch gelten die hierbei entwickelten Strukturen auch im Bereich der "Gesetzgebung“. Bevor jedoch dieser Brückenschlag der Gemeinsamkeiten vollzogen wird, sei hier noch von einer eigenartigen Erscheinung die Rede, die es erst verständlich macht, warum die Massenlinie auch im Gesetzgebungsverfahren Eingang finden konnte: Der Normadressat wird in China nämlich unter dem Bild des Guerillakämpfers gesehen. Das will nichts anderes besagen, als daß die Taktik lokaler operativer Autonomie unter zentraler Kursbestimmung, wie sie gemeinhin für selbständig ausschwärmende Partisaneneinheiten maßgebend ist, auch auf die maoistische Normsetzungspolitik ihren Einfluß ausübt.

Dieses Denken in den Kategorien des Partisanenkrieges darf nicht weiter überraschen, wenn man bedenkt, daß die Kommunisten bis zu ihrem Sieg im Jahre 1949 immerhin 22 Jahre lang unter den Bedingungen des täglichen Kleinkriegs gegen die Kuomintang und später gegen die Japaner gelebt haben. Während dieser zwei Jahrzehnte, die nicht von ungefähr die Lebensauffassung der meisten heute führenden Kommunisten geprägt haben, galt es, in weit auseinandergezogenen Fronten, die obendrein im Rücken des Feindes verliefen, oft wochenlang auf sich selbst gestellt, mit den Anforderungen der jeweiligen Situation fertig zu werden. Die Führung in Yenan mußte sich darauf beschränken, die großen Linien vorzuzeichnen, war aber im übrigen darauf angewiesen, daß dieses magere Befehlsskelett von den lokalen Verbänden mit Leben gefüllt würde.

Wichtigste Voraussetzung für eine erfolgreiche Arbeitsteilung zwischen „Gesetzgeber" und "Gesetzesadressaten" war selbstversändlich eine Atmosphäre politischer (sprich: ideologischer) Gemeinsamkeit, die vor allem während des Krieges gegen Japan durch innerparteiliche "Ausrichtungskampagnen" und nicht zuletzt natürlich durch die ständige Nähe des Feindes garantiert wurde. Nicht der bürokratisch versierte Routinier wurde damals als tauglich empfunden, sondern vielmehr der „Kader“, der weniger befahl und instruierte als vielmehr durch seine Begeisterungsfähigkeit, seine Überzeugungskunst und durch das permanente Definieren von Gruppenzielen ein gemeinsames Ethos zu erzeugen verstand.

Dieser Typ von Kader, dessen Vokabular durch und durch militärisch geprägt war, darf als die chinesische Antwort auf den „Beamten“ angesehen werden: Gelten für diesen (nach der Weberschen Kategorientafel) Arbeitsteilung, Spezifizierung von Rollen, Trennung von befehlserteilenden, befehlsübermittelnden und ausführenden Funktionen sowie eine rein fachmännische Orientierung als Ideal, so nimmt der chinesische Kader demgegenüber mit seiner grundsätzlichen Allzuständigkeit, mit seiner Angewohnheit, die Rollen aus ihrer spezifischen Vereinzelung zu lösen, befehlende und ausführende Funktionen ineinander verfließen zu lassen und der Politik vor dem Expertentum den Vorrang einzuräumen, eine Art Antipodenstellung ein ${ }^{87}$.

In den frühen fünfziger Jahren, als das sowjetische Vorbild eine völlig neue Wertlandschaft zu schaffen schien, hatte diese Konzeption kaum Chancen. Im Rahmen des ersten Fünfjahresplans vor allem war der Fachmann gefragt, der

86 Vgl. z. B. Summary of World Broadcasts, Part 3, The Far East FE 3253 B 7

87 Shurmann a. a. O., S. 220-238. 
es verstand, eine komplizierte Planpause zu erstellen und sie auf die chinesische Wirklichkeit zu übertragen. In der von Detailfragen beanspruchten Bürokratie war während dieser Jahre wenig Zeit für „Politik“. Das alte Kaderideal schien angesichts dieser modernistischen Strömungen der Vergangenheit anzugehören. Erst mit der Renaissance der Ideologie während der Zeit der „Drei Banner“ (1958 ff.) und insbesondere während der Kulturrevolution (1966 ff. $)^{88}$, als der Maoismus seine Wiederauferstehung feierte, besann man sich auf die alten Ideale, vor allem, nachdem der Kaderapparat und damit das personelle Substrat jener modernistischen Strömungen weitgehend zerstört worden war.

Die maoistische Politik war aber nicht nur auf Zerstörung des „verbürgerlichten“ Machtapparates aus, sondern suchte zugleich eine Atmosphäre zu schaffen, die jener der Partisanenzeit nicht unähnlich war. An die Stelle des äußeren Feindes wurde der innere Feind mit den Mitteln des Klassenkampfes und der Klassenanalyse neu aufgebaut. Zugleich setzte der Appell ein, sich auf den „Krieg vorzubereiten" 89 .

Diese „Kriegsvorbereitungspolitik“ schlug sich später vor allem in einer weitgehenden Dezentralisierung nieder, die aus China nicht nur eine Zellkolonie unangreifbarer Einheiten machen sollte, sondern auch eine Verwaltungsstruktur schuf, die ganz im Geiste der "Massenlinie“ möglichst nach unten verlagert und damit in ein Raster von Verwaltungsmonaden aufgelöst wurde, deren Koordinierung durch das gemeinsame Bekenntnis zur "Linie Mao Tse-tungs" idealiter garantiert werden soll, mindestens aber durch den in der Form freundlichen, in der Sache aber harten Zugriff der Volksbefreiungsarmee gesichert wird.

Mit der Parusie einer, wenn nicht wirklichen, so doch zumindest fingierten Situation des Partisanenkrieges (Feinde von innen und außen, Dezentralisierung, Autarkisierung) ist am Ende der sechziger Jahre eine Atmosphäre geschaffen, in der die alte Kadertradition wieder Wurzeln fassen kann. Die Funktionäre, die heute in den Revolutionskomitees mitarbeiten, sind zumeist nach dem alten Modell neu geformt worden und haben sich laufend (durch Schulungskurse, „7.-Mai-Schulen“, Mitarbeit in der Produktion, Einfachheit und Erziehungsbereitschaft) als getreue Anhänger der "Linie des Vorsitzenden Mao“ zu bewähren. Insbesondere haben sie sich die "drei großen Arbeitsstile“ (Verbundenheit von Theorie und Praxis, Verbundenheit von Kadern und Massen, Kritik und Selbstkritik) zu eigen zu machen. Vor allem die Verbundenheit von Kadern und Massen wirkt sich dahin aus, daß die Unterschiede zwischen beiden (mindestens der Theorie nach) immer stärker verschwinden. Letzten Endes ist also jeder Angehörige der "Massen“ ein Kader oder hat sein Verhalten so einzurichten, als ob er einer von ihnen wäre ${ }^{90}$.

Diese neue (bzw. erneuerte) Auffassung vom Befehls- und Normadressaten konnte nicht ohne Einfluß auf die "Gesetzgebung“ bleiben. In der Tat verloren die „Rechtsnormen“ im Laufe der Kulturrevolution immer mehr von ihrem spezi-

88 Eigentlich schon mit der "Sozialist. Erziehungsbewegung “ (1962 ff.).

89 Ausdrücklich als eine der 5 Hauptaufgaben für das Jahr 1968 hervorgehoben (vgl. VZ. 1. I. 1968).

90 In der Periode der Kulturrevolution ließen sich die „Massen“ in eine gesellschaftliche Matrix von sechs sozialen Gruppen auflösen, nämlich Arbeiter, Bauern, Soldaten, technische und administrative Kader, Intelligentsia und Studenten (vgl. Stuart Kirby "The Framework of the Crisis in Communist China ${ }^{\alpha}$, Current Scene 1968, Nr. 2). Jeder dieser Rollenträger soll sich eine Haltung anerziehen, die ihn nicht als virtuosen Solisten seines Fachs erscheinen läßt, sondern als einen Könner, der sämtliche Instrumente zu spielen versteht. Der Student darf also sein Ziel nicht nur darin sehen, später zur Intelligentsia aufzurücken, sondern er hat auch Bauer zu sein, indem er immer wieder auf dem Lande mitarbeitet, Soldat, indem er entweder direkt der VBA angehört oder aber der Miliz; Arbeiter, indem er sich immer wieder in den Arbeitsprozeß bei den Fabriken einschaltet und auch Funktionär. Man könnte hier von einem das gesamte gesellschaftliche Leben durchwaltenden Prinzip der sozialen Angleichung sprechen. 
fisch juristischen Beigeschmack: Sie ergingen nur noch in großen Linien, so daß den einzelnen Untereinheiten ein weiter Verwirklichkeitsspielraum bleibt. Insofern gleicht jede Abteilung einer Guerilla-Einheit, die ungewissen Situationen durch rasch entschlossenes Handeln begegnet und jedes bürokratische Verweilen, Subsumieren und Abwägen als Zeitverlust und bürokratische Schwerfälligkeit empfindet.

Ein Normadressat, wie er von der neuen Gesetzgebung vorausgesetzt wird, ist nicht denkbar, ohne eine grundsätzlich neuartige Auffassung von der ganzen Organisationsstruktur, in die dieser Adressat eingebettet ist. Stillschweigend geht deshalb die "Legislative“ von der Voraussetzung aus, daß die Merkmale des Ausschußprinzips und der vertikalen Dekonzentration auch im Bereich der Gesetzgebung gelten. Die Norm wendet sich also nicht mehr an die fachlich jeweils „zuständigen Behörden“, sondern an die Revolutionskomitees, die das gesamte Spektrum verschiedenartigster Zuständigkeitsbereiche in sich zusammenfassen. Da überdies der Wald der Behörden in den höheren Instanzen während der Kulturrevolution weitgehend gelichtet wurde, ist auch im vertikalen Bereich die Aufgabe der "Gesetzesausfüllung und -ausführung“ fast ausschließlich den Revolutionskomitees zugefallen.

Damit aber hat die "Massenlinie “ auch in die Gesetzgebung Eingang gefunden, allerdings auf Kosten einer Art Militarisierung des Gesetzgebungsverfahrens. Man könnte sogar von einem „hsia fang“ der Gesetzgebung sprechen ${ }^{91}$.

\section{Das Recht und die maoistische Konzeption der Praxis}

Auch die grundsätzlich praxisorientierte Verhaltensweise der chinesischen Kommunisten fand in die "Gesetzgebung " Eingang"2.

Die maoistische Erkenntnistheorie läßt die "Erkenntnis“ in drei Stufen zustande kommen: Auf die sinnliche Rezeption (1. Stadium) folgt die begriffliche Verarbeitung (2. Stufe). Die nunmehr gewonnenen Urteile und Schlußfolgerungen müssen sodann in die Praxis zurückgefüttert werden, um sich dort zu verifizieren (3. Stufe). Es genügt also nicht, daß man die Welt nur rational versteht und theoretisch interpretiert. Die Theorie ist vielmehr, wie es ja bereits in den Feuerbach'schen Thesen steht, wieder auf die Praxis anzuwenden. Praxis aber bedeutet Kampf, und Kampf Veränderung. Der Erkenntnisprozeß beginnt bei der Praxis und kehrt wieder zu ihr zurück. Die Praxis also ist es, die zum eigentlichen Kriterium der maoistischen Erkenntnistheorie wird ${ }^{93}$.

91 „hsia-fang“ (wörtlich: „Hinunterschicken“) ist ein Ausdruck, der all jene Vorgänge umschreibt, bei denen Kader zur Produktionsarbeit oder Städter aufs Land verschickt werden. Hsia-fang ist nach Ansicht der Maoisten die beste Methode, um die drei im Text erwähnten "großen Arbeitsstile“ in den betroffenen Individuen zu erneuern. "Hsia-fang ${ }^{\alpha}$ der Gesetzgebung hieße also nichts anderes, als daß Gesetze nicht mehr am grünen Tisch gemacht, sondern möglichst "weit unten “ und möglichst mitten im Getriebe des Geschehens Gestalt annehmen.

Zur "Massenlinie“ im Rechtssystem vgl. i. ü. Cheng P'u „Thoroughly destroy the old Legal System and eliminate Bourgeois Legal Thought" in ",jasp“, "Chinese Law and Government" vol. I, Nr. 3, Fall 1968, S. 77: Cheng betont auch besonders deutlich den Überbaucharakter des Rechts, das der sozialist. Basis angeglichen werden müsse a. a. O. 68, 72, 76 (Urspr. in Cheng-fa yen-chiu 1964, Nr. 2).

92 Zum Empirismus im chin. Recht vgl.:

a) David C. Buxbaum "Preliminary Trends in the Development of the Legal Institutions of C. C.", in

"Government of C. C. “" ed. by G. P. Jan, San Francisco, Calif., 1966, S. $343 \mathrm{ff}$.

b) Stahnke, der eingehend die Entstehung des StGB schildert, a. a. O. $519 \mathrm{ff}$.

c) Hsiang Shih „New Problems in the Realm of Legal Studies“, in „jasp“, Chinese Law and Gov., vol. I.,

Nr. 2, Summer 1968, S. 3 ff.

93 „Uber die Praxis“, in: Tilemann Grimm, „MTt, Ausgew. Werke“ Fft 1964, S. 34, 40, 42. 
Es versteht sich von selbst, daß eine so eindringlich vorgetragene und von den Kommunisten seit dreißig Jahren bewußt durchexerzierte Maxime nicht ohne Einfluß auf die Gesetzgebung bleiben konnte. Je „chinesischer" der Kommunismus wurde, desto nachhaltiger prägten sich drei juristische Konsequenzen aus:

a) Formelle Gesetze, die unter dem harten Zugriff der Systematik eine Rechtsmaterie allzufrüh präjudizieren könnten, sind schon seit Mitte der fünfziger Jahre ganz weggefallen. Die Ursache für diesen Abbau ist nicht darin zu erblicken, daß der Nationale Volkskongreß seit 1964 nicht mehr zusammengetreten ist. Vielmehr verlief der Kausalitätsprozeß in umgekehrter Richtung. Gerade weil die Aufgaben des Kongresses in anderen Instanzen erledigt oder aber vom Sog der allgemeinen institutionellen Entwicklung absorbiert wurden, verlor der Kongreß seine Existenzberechtigung. Die maßgebende juristische Zeitschrift der Volksrepublik China bezeichnet es in der Tat als geradezu „verrückt" ${ }^{\text {"94 }}$, tatbestandlich genau umschriebene Kodifikationen in die Welt zu setzen; sei „doch die objektive Wirklichkeit viel zu kompliziert, zu sehr im Fluß und ständig dem Wechsel unterworfen".

Nur generalklauselartige - und daher flexible - Rechtsvorschriften könnten den Wettlauf mit der Wirklichkeit bestehen.

Die reiche Gesetzgebungsarbeit der Volksrepublik, die sich insbesondere zu Beginn der fünfziger Jahre nachweisen läßt, kann nicht als Gegenargument gewertet werden; denn erst in der zweiten Hälfte der fünfziger Jahre löste sich China langsam aus der Vormundschaft der Sowjetunion und begann seinen eigenen Weg zu gehen.

b) Mit der oben angedeuteten Generalklauseltechnik ist bereits ein weiteres Prinzip der chinesischen Gesetzgebungsarbeit gewonnen: Vorschriften dürfen nämlich nie zu eng sein und sollen deshalb nur den allgemeinen Rahmen abstecken, um der Vielfalt sozialer Wirklichkeit genügend Spielraum zu lassen. Besonders bedenklich prägt sich diese Tendenz zur generalklauselartigen Weite im Strafgesetzbuch aus. Artikel 18 des Gesetzes über die Bestrafung konterrevolutionärer Aktivitäten lautet z. B.: „Dieses Statut ist auch anwendbar auf konterrevolutionäre Verbrechen, welche ... 95“

c) Gesetze sind deshalb auch nicht als Garanten der Rechtssicherheit gedacht, deren gleichmäßige Anwendung durch geschultes Richterpersonal mit Hilfe strenger Interpretation und durch „ständige Rechtsprechung“ verfestigt wird. Überhaupt wären rechtstechnische Kunstgriffe, wie die Hilfsmittel der historischen oder systematischen Gesetzesauslegung im sino-juristischen Bereich ganz fehl am Platze; denn ein "Gesetz" kann nach chinesischer Auffassung nicht Geltung kraft seiner bloßen Existenz ${ }^{96}$ beanspruchen, sondern wird lediglich als unselbständiger Bestandteil einer kommunistisch interpretierten Gesellschaftsordnung verstanden und borgt sich von diesem größeren Bezugsrahmen her seine Legitimität.

Leben erhält die Rechtsvorschrift erst durch ihre konkrete Verwirklichung in einer konkreten Situation. Dieser Verifizierungsprozeß ist nicht nur das Ergebnis einer sauberen juristischen Subsumption, sondern führt - richtig vollzogen $\mathrm{zu}$ einer harten Auseinandersetzung mit den Kräften, die einer revolutionären

\footnotetext{
94 Cheng-fa yen-chiu, April 1958, zit. in „China News Analysis“ (fortan CNA) Nr. 184, S. 2, ähnlich Mao, "Praxis ${ }^{\alpha}$, bei T. Grimm a. a. O. 43.

95 Stahnke a. a. O. (FN. 16), S. 514.

96 Zur rechtsphilosophischen Geltungslehre, vgl. Radbruch „Rechtsphilosophie“ a. a. O., S. $174 \mathrm{ff}$.
} 
Rechtsetzung Widerstand leisten. „Revolutionäre Rechtsetzung“ ist also letzten Endes nichts anderes als eine ziemlich pauschal gefaßte Anweisung zum Klassenkampf.

Wer garantiert freilich, daß solche klassenkämpferischen Auseinandersetzungen, die ja in der Regel nicht unbedingt sachlich verlaufen, zu Ergebnissen führen, die mit dem "Gesetzes"zweck intendiert sind? An dieser Stelle ist nun eine weitere Prämisse einzuführen, die mit zum eisernen Bestandteil des Maoismus gehört: nämlich die Überzeugung, daß den „Volksmassen“ eine unbegrenzte Schöpferkraft innewohnt ${ }^{97}$ und daß sie, wenn ihnen nur einmal die vom „Klassengegner" angelegte Binde von den Augen genommen wird, zur vollkommenen Selbsterziehung im sozialistischen Geist fähig seien. Getragen von einem „gewaltigen Drang zum Sozialismus"98 werden sie deshalb im Wege des Klassenkampfes, bei dem sie wirklich ernsthaft gefordert werden, zumindest in die Wahrheit hinein „stolpern" $"$ 90.

"Sachlichkeit" und richterliche Objektivität sind nach kommunistischer Auffassung letzten Endes Bestandteile des Überbaus: Sozialistische „Sachlichkeit" und „Sachlichkeit" im herkömmlichen Sinne haben deshalb nur die Worthülse, nicht jedoch den Inhalt gemeinsam. Das Endergebnis einer Gesetzesanwendung ist deshalb, wenn es den realen Bedürfnissen der Massen entspricht ${ }^{100}$, stets richtig, auch wenn das Anwendungsverfahren einem außenstehenden Beobachter nicht ganz geheuer ist.

Unter den oben angeführten drei Besonderheiten hat die Scheu gegenüber systematischen und ausführlichen Kodifikationen in der chinesischen Rechtsgeschichte wohl die längste Tradition. Die Kommunisten kehren mit ihrer Praxis also sozusagen zum chinesischen Normalzustand zurück, der nur durch vereinzelte $\mathrm{Ge}$ setzgebungswerke ${ }^{101}$ und vor allem durch die umfassende Rezeption europäischen Rechts während der Kuomintangzeit gewisse Ausnahmen erlitt: Mit dem Ende der Kuomintang kam auf dem Festland auch zugleich eine wichtige Periode der chinesischen Rechtsgeschichte zum Abschluß102.

Bei den Kommunisten war die Gesetzgebung eine Zeitlang zwar genauso produktiv wie bei ihren Vorgängern, doch änderte sich diese Praxis im Laufe der Jahre immer mehr. Vor allem die Gesetzgebungstechnik machte einen grundsätzlichen Wandel durch: Waren die Rechtsnormen insbesondere Mitte der fünfziger Jahre noch ziemlich ausführlich und lückenlos, so wurden sie nach 1958 inhaltlich immer unpräziser.

Damit aber wurde ein weites Feld für „Gesetzesauslegungen“ und „Rechtsfindung" geschaffen. Die Rechtsadressaten wurden immer mehr zur schöpferischen Mitwirkung am legislativen Entfaltungsprozeß aufgerufen. Da den Vollstreckern des "Gesetzes" im allgemeinen keine genauen Interpretationsmaßstäbe an die Hand gegeben wurden, war dieser Schöpfungsprozeß keine einfache Aufgabe. Eines jedoch konnte als sicher gelten: Selbst wenn man mit so genauen Methoden wie den im deutschen Recht geltenden subjektiven und objektiven Auslegungsmaß-

97 „Worte des Vorsitzenden Mao Tse-tung ${ }^{\alpha}$, Peking 1967, S. 140

98 „Worte des Vorsitzenden Mao Tse-tung “, Peking 1967, S. 143.

99 Analog zu dem engl. Rechtsausdruck ,stumbling into wisdom“, vgl. Radbruch G. „Der Geist des englischen Rechts“, Göttingen 1958, 4. Aufl. S. 64

$100,90 \%$ der Massen ${ }^{\alpha}$ (!) vgl. MTt AW III S. 446, 283, 281.

101 Die wichtigsten Modifikationen sind in ihrer historischen Reihenfolge zusammengestellt bei Joseph Needham "Science and Civilisation in China ", Cambr., Engl. 1956, S. 521-524.

102 Zur Abschaffung der KMT-Gesetze durch die Kommunisten, vgl. FN. 108. 
stäben herangeht ${ }^{103}$, landet man doch immer wieder bei der marxistisch-leninistischen Theorie, die letzten Endes die Lücken zu füllen hat; denn gerade in einer Gemeinschaft wie der chinesischen ist der Richter, der Verwaltungsbeamte oder aber der einzelne an die in der Gesellschaft herrschenden und an die im "Gesetze“ schlummernden Werturteile gebunden.

Er hat die Lösung aus dem Geist des "Gesetzes“ zu finden, der in jedem Falle kommunistisch bestimmt ist, sei es nun, daß man bei der Auslegung vom „Willen des Gesetzgebers“ ausgeht (subjektive Theorie) oder aber die „gegenwärtige Interessenlage" maßgebend sein läßt (objektive Theorie).

Die juristische Struktur einer Rechtsnorm wird also immer mehr von der Ideologie überwuchert, so daß man wohl zu Recht von einer Herrschaft außerjuristischer Gesichtspunkte im jüngeren chinesischen Rechtssystem sprechen darf.

Das alte Tauziehen zwischen "li“ und „fa“ taucht damit unter moderneren Gesichtspunkten wieder auf ${ }^{104}$.

Lange Zeit waren im alten China die normativen Ordnungen religiöser, ethischer und gewohnheitsrechtlicher Observanz ungeschieden. Diese Periode einer einheitlichen und ungeteilten Lebensordnung endete im wesentlichen erst mit dem Zeitalter der Legisten ${ }^{105}$, deren staatsphilosophische Spekulationen sich in ersten Kodifikationen niederschlugen.

Die neuen Strömungen waren insofern revolutionär, als Recht, Gesetz und Befehl von oben gleichgesetzt wurden. An die Stelle der Obervanzen traten die Befehle der Regierung. Die Gesetze blieben abstrakt abweisend, gaben in ihrer Wortkargheit keine Begründung und forderten von dem Rechtsunterworfenen passiven Gehorsam gegenüber der Obrigkeit. Auch führten sie - in einer hierarchischen Gesellschaftsordnung! - das Prinzip der Gleichheit vor dem Gesetz ein ${ }^{106}$. Gegen diese Neuerung erhob sich schon bald der Widerspruch der Konfuzianer, die zu bedenken gaben, daß Gesetze nur zum Prozessieren, zu Wortklaubereien und Streitereien führen könnten. Der Weg zu Anstand und Sitte (li) werde durch ein Blatt Papier versperrt. Greife nicht gerade dort die Lust am Reglementieren um sich, wo Dekadenz und innerer Zerfall schon am weitesten gediehen seien? ${ }^{107}$.

Kein Wunder, daß während der Han-Dynastie schon bald die Konfuzianisierung und Ethisierung des Gesetzes begann: „li“ löste nach und nach das positiv geregelte „fa“ $\mathrm{ab}$.

103 In der deutschen Rechtswissenschaft haben sich verschiedene Meinungen herausgebildet, ob man den Inhalt des gesetzgeberischen Willens durch Zurückgehen auf die Zeit der Entstehung des Gesetzes ermitteln soll (so die subjektive Theorie) oder aus den Vorstellungen und Werturteilen der Gegenwart heraus (so die objektive Theorie).

a) Die subjektive Theorie fragt: „Was hat ein Gesetz, das die $\mathrm{d}$ a mals von ihm angeschaute Interessenlage so geregelt hat, damit vernünftigerweise bezweckt, welche Werturteile der damaligen Kulturgemeinschaft sind damit zum Ausdruck gebracht? ${ }^{\star}$

b) Die objekt ive Theorie löst den Wortlaut von den Vorstellungen der am Gesetzeserlaß beteiligten Personen und fragt: "Was kann ein Gesetz, das angesichts der g e g e n w är t i g e $n$ In e r e ss e n l a g e eine solche Regelung trifft, damit vernünftigerweise bezwecken; welche Werturteile der gegen-
wärtigen Kulturgemeinschaft finden darin Ausdruck? ${ }^{\alpha}$ (vgl, dazu Heinrich Lehmann ${ }^{*}$ Allgemeiner Teil wärtigen Kulturgemeinschaft finden darin Ausdruck? ${ }^{\alpha}$ (vgl.
des Bürgerlichen Gesetzbuches“, 8. Aufl., Berlin 1954, S. 53 f.).

104 Es ist hier nicht der Ort, genauere Ausführungen über diese beiden Begriffe der chinesischen Rechtsphilosophie zu bringen. Sehr allgemein läßt sich "fa $a^{\alpha}$ aber mit "positivem Recht ${ }^{\alpha}$, "li ${ }^{\alpha}$ dagegen mit „Sitte ${ }^{\alpha}$ übersetzen.

105 Erste Ansätze um 400 v. Chr.: Ein gewisser Tzu Ch'an schlägt im Staate Ching die erste Gesetzestafel an. Am konsequentesten bildete sich die Methode, mit Gesetzen zu regieren, im Staate Ch'in (221-206 v. Chr.) heraus, dessen Praktiken eine Apotheose auf die Lehre der Legisten (Fa-Chia) waren.

106 Die obigen Ausführungen verdankt der Autor zum Teil einer Vorlesung von Karl Bünger (am 9. 6. 1969 an der Universität Hamburg).

107 Nachweise u. a. bei Needham a. a. O. S. 522. 
Eine Parallele zu dieser traditionellen Entwicklung zeigt sich im heutigen kommunistischen China: Es baute das Recht der Kuomintang ab ${ }^{103}$ und ist im Laufe seiner zwanzigjährigen Entwicklung immer mehr dazu übergegangen, noch bestehendes „fa“ $\mathrm{zu}$ „maoisieren“ und damit juristisch $\mathrm{zu}$ entwerten. Damals wie heute büßte die Rechtsordnung ihre Eigenexistenz ein und kehrte wieder in den Schoß der allgemeinen gesellschaftlichen Normen zurück. Kein Wunder deshalb, daß die Gesetzgebung von dieser Entwicklung so sehr affiziert wurde, daß ihr Aussehen im Jahre 1969 mit den Erscheinungsformen von 1954 fast keine Gemeinsamkeiten mehr aufweist.

Doch zurück zur Gegenwart.

Wie das praxisorientierte - und auf die „Massenlinie“ eingeschworene „Gesetzgebungs"-Verfahren funktionieren soll, zeigt ein Artikel der Shanghaier Wenhui-Zeitung, die sich rühmen darf, das Denken Mao Tse-tungs immer schon am treuesten abgespiegelt zu haben ${ }^{\mathbf{1 0 9}}$ :

In einer Shanghaier Maschinenfabrik sollten Regelungen über Budget-Fragen, Warenlager, Produktionsüberwachung, Qualitätskontrolle und über einige „soziale Angelegenheiten, wie Service-Trupps, Klinik- und Massageeinrichtungen, sowie über neue Formen des Managements" erlassen werden. Ein „ArbeiterForschungsteam" ${ }^{110}$ und selbstverständlich das Revolutionskomitee der Fabrik waren es, die sich dieser Aufgabe besonders annahmen. Beide Gremien sollten freilich nicht aus eigener Machtvollkommenheit handeln, sondern sich nur als Mentoren fühlen. Zwei Methoden machten sie sich bei ihrer "gesetzgeberischen" Arbeit vor allem zu eigen, nämlich die Maxime des "Lernens von den Massen“ und des „Experimentierens“. $\mathrm{Zu}$ diesem Zweck hielten sie mehrere Massendebatten $a b$, bei denen sich Gelegenheit ergab, die Erfahrungen der Arbeiter zusammenzufassen. Die dabei gewonnenen Gesichtspunkte wurden systematisiert, jedoch nicht sofort verkündet, sondern nur in zwei Werksabteilungen längere Zeit getestet. Während dieser Versuchsperiode hielt das „Arbeiter-Forschungsteam" wiederum mehrere Versammlungen zur „revolutionären Massenkritik“ $a b$, bei denen die Arbeiter ihre Meinungen frei äußern und die Kompilatoren zur kritischen Überprüfung ihres Entwurfs veranlassen sollten. Anschließend wurden die Satzungen erneut überarbeitet und waren dann offensichtlich für die Verkündigung reif. Diskussionen und Massenversammlungen sollen jedoch auch künftighin dafür sorgen, daß Kritik und klassenkämpferische Gesinnung nicht von der einmal fixierten Regelung hinwegmanipuliert werden.

Obwohl sich der Klassenkampf im vorliegenden Fall an der trockenen Materia offensichtlich nicht recht entzünden konnte, wird er doch als das eigentlich beseelende Element auch im „Gesetzgebungsverfahren“ postuliert"11. Damit taucht ein weiterer Gesichtspunkt auf, der im nächsten Abschnitt zu behandeln ist.

108 Das Gesetzgebungswerk der KMT (Die sog. „6 Gesetzbücher“ liu fa wurde von den Kommunisten 1949 durch 3 sukzessive Maßnahmen abgeschafft:

a) Im Februar 1949 erließ das ZK eine entsprechende Direktive.

b) Am 1. April 1949 verkündete die nordchin. Volksregierung ein Dekret gleichlautenden Inhalts. c) Am 29. Sept. 1949 schließlich stimmte die Polit. Konsultativkonferenz gleich in ihrer ersten Plenarsitzung abermals und endgültig für die Abschaffung (vgl. Chou Hsin-min in SCMP a. a. O. 5). Zur Abschaffung vgl. auch Karl Bünger "Die Rezeption des Europäischen Rechts in China ${ }^{\alpha}$, aus

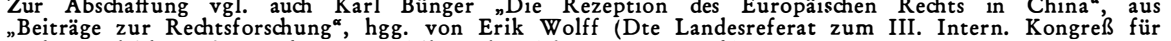
Rechtsvergleichung in London 1950, Teil I, Bln.-Tüb. 1950, S. 166 f.).

109 Abgedr. in Summary of World Broadcasts (SWB), Part. III, The Far East Nr. 3198, B. II, S. 11 ff.

110 bestehend aus Arbeitern, Kadern und „revolutionären Technikern ${ }^{\star}$, ib.

111 ib. 11. 


\section{Das Recht und die Theorie der Widersprüche}

„Hauptzweck“ einer sozialen Gesetzordnung muß es sein, „die Feinde zu unterdrücken und das Volk zu schützen"112.

Mit dieser klassengebundenen Zieldefinition, die von der amtlichen Pekinger Volkszeitung abgedruckt wurde, klingt ein Leitthema an, das die Schriften Maos von Anfang bis Ende durchzieht ${ }^{113}$, nämlich die ständige Betonung von Klassenkampf und Klassen wi d e r s p r ü c he n.

Hinlänglich bekannt sind die Kriterien, nach denen Mao die Gesellschaft in Volk und „Nicht-Volk“ einteilt: Die „Widersprüche im Volk“, die mit Hilfe demokratischer Methoden (Überredung, Kritik, Diskussion und Erziehung) lösbar sind und die "Widersprüche zwischen uns und unseren Feinden“, die als „kontradiktorisch" gelten und deshalb mit dem eisernen Besen der Diktatur des Proletariats ausgekehrt werden müssen, sind zwei völlig verschiedene Kategorien, die scharf auseinandergehalten werden müssen und die bei jeder Klassenanalyse anzuwenden sind ${ }^{114}$.

Auch das Recht hat sich an dieser Zweiteilung zu orientieren und als ein Instrument zu dienen, das beide Arten von Widersprüchen mit jeweils adäquaten Mitteln lösen soll115.

a) „Widersprüche im Volk“ sind solche Konflikte, die „aus der Gleichartigkeit von grundlegenden Interessen erwachsen "116. Rechtsadressaten vor allem,

112 Chou Hsin-min "Law is a sharp weapon of class struggle , nach VZ. 28. X. 1964, SCMP Nr. 3339 , S. 3.

113 Schon sein erster in den AW abgedruckter Aufsatz "Analyse der Klassen in der chinesischen Gesellschaft ${ }^{\alpha}$ vom März 1926 läßt den Gedanken der Klassenwidersprüche anklingen und hebt vor allem die Frage nach dem Feind hervor (MTt AW I Peking 1968, S. 9 ff.). Den Höhepunkt dieser Entwicklung bildet die Widerspruchsrede von 1957, abgedruckt bei Tilemann Grimm, a. a. O. S. $78 \mathrm{ff}$.

114 ib. S. 88, 98. Zu den drei Auswirkungen der W'iderspruchsdoktrin auf das Rechtsgefüge vgl. Jyun-hsyong $\mathrm{Su}$, "Wesen und Funktion von Staat, Recht und Regierung im Kommunist. China ${ }^{\alpha}$ Osteuropa-Recht 1969, Nr. 3, S. 162; ferner Buxbaum a. a. O. 347.

115 Wenn J. H. Su die chinesischen Rechtsnormen wegen ihrer „Klassengebundenheit und ideologischen

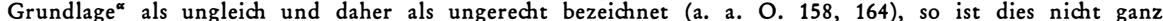
zutreffend. Es ist zwar richtig, daß die Gleichheit, die gebietet, Gleiches gleich, Ungleiches aber nach den konkreten Umständen ungleich zu behandeln, das Kriterium für die Gerechtigkeit abgibt. Eine ganz andere Frage jedoch ist es, nach we $1 \mathrm{ch}$ e $\mathrm{n}$ Maßstäben die Gleichheit definiert wird (vgl. Radbruch, Rechtsphilosophie a. a. O., S. 147-155: „Individualwerte, Kollektivwerte und Werkwerte ${ }^{\alpha}$ werden dort als die drei möglichen Orientierungskriterien der Gleichheit anerkannt). Ein Staatswesen, das die individuelle Persönlichkeit als höchsten Wert betrachtet, wird a 11 e Menschen vor dem Gesetz gleichstellen. Eine Gesellschaft andererseits, der es vor allem um Gemeinschaftswerte zu tun ist, mag jene Kollektive als gleichwertig erachten, die der Gesamtgesellschaft nach Maßgabe der ideologischen Ausrichtung adäquat sind. Eine kommunistisch konzipierte Rechtsauffassung, die „alle Proletarier ${ }^{\alpha}$ (seien sie es der Geburt oder aber der Gesinnung nach) als gleich wertvoll, alle Nichtproletarier aber als Feinde behandelt, verbleibt demnach im Rahmen der "Gerechtigkeit ${ }^{\alpha}$, wenn sie nur zwei Bedingungen erfüllt:

a) sie hat alle "Proletarier ${ }^{\alpha}$ wirklich gleich und alle Nichtproletarier wirklich nach den jeweiligen Umständen ungleich zu behandeln. Auf diese Radbruchsche Konzeption ist besonders deshalb hinzuweisen, weil gerade Su seinen Gerechtigkeitsbegriff auf Radbruch stützt (a. a. O., S. 158 und a. a. O., Fußnote 17).

b) Sie hat (und hier ist ein materieller Maßstab anzulegen) ferner die Würde des Menschen zu achten, die immer dann als verletzt anzusehen ist, wenn der Mensch nicht als Persönlichkeit, sondern als Sache (als "Untermensch ${ }^{\alpha}$ ) behandelt wird (Radbruch "Gesetzliches Unrecht und gesetzliches Recht ${ }^{\alpha}$ abgedruckt in "Rechtsphilosophie ${ }^{\alpha}$ a. a. O., S. 354). Liegt ein solcher Verstoß vor, so handelt es sich nicht etwa um „unrichtiges“ (weil ungleiches:) Recht; vielmehr liegt dann überhaupt kein „Recht ${ }^{\alpha}$ vor (Radbruch a. a. O. 354).

Unter diesen zuletzt genannten Gesichtspunkten könnte also weiter gefragt werden, ob das chinesische Recht die "Feinde des Volkes ${ }^{\alpha}$ wie "Nichtmenschen" behandelt. Aus dem Wortlaut der "Rechtsnormen" läßt sich ein solcher Verstoß wohl kaum herleiten.

In der Rechtspraxis andererseits werden diese "Feinde ${ }^{\alpha}$ zwar manchmal mit semantischen Mitteln in die Kategorie von Nichtmenschen eingestuft (vgl. dazu insbesondere H. C. Chuang "The Great Proletarian

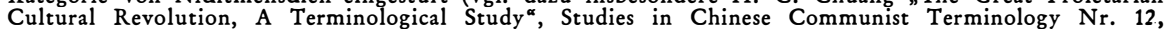
Berkeley/Calif. 1967, S. 17-26), doch zeigt sich immer wieder, daß gerade in der Volksrepublik China eine weite Kluft zwischen Worten und Taten herrscht. Selbst wenn aber das eine oder andere chinesische Gesetz nach den oben aufgezeichneten Kategorien als „Nicht-Recht ${ }^{\alpha}$ zu gelten hätte, so bliebe doch die übrige Rechtsordnung unberührt.

Im übrigen führt die ganze Frage hier zu weit vom Thema ab; denn die vorliegende Abhandlung geht ja gerade von der Voraussetzung aus, daß es sich bei den chinesischen Normen um eine Rechtsordnung handelt.

116 Min-fa chi-pen wen-t'i, a. a. O., S. 6. 
die den sozialistischen Eigentumsbegriff, die volksdemokratische Diktatur und den demokratischen Zentralismus billigen, gehören folglich $\mathrm{zu}$ einer solchen sozialistischen Interessengemeinschaft ${ }^{117}$.

Wer den „sozialistischen Aufbau“ also grundsätzlich bejaht, aber wegen technischer Kleinigkeiten mit seinen Rechtsgenossen in Streit gerät, ist mit den "demokratischen" Methoden der Erziehung und Überredung anzufassen. Hierbei werden vor allem außerjuristische Maßnahmen zum Zuge kommen, insbesondere „Versammlungen zur Kritik und Selbstkritik“, „Mao-Tse-tung-Kurse“ und dgl.

b) „Widersprüche zwischen uns und unseren Feinden“ offenbaren sich umgekehrt darin, daß bestimmte Personengruppen für sich subjektive Rechte beanspruchen, also etwa auf ihr Eigentum pochen oder die Einhaltung solcher obligatorischer Verpflichtungen verlangen, die ausschließlich dem individuellen Eigennutz dienen.

Manifestationen dieser Art gelten als Rückstände „feudaler“ Denkgewohnheiten und zeugen vom spirituellen Fortbestehen der alten „Klassen“, die ihrer äußeren Erscheinung nach doch längst untergegangen sind. An dieser Stelle nun hat das Recht mit seiner ganzen Schärfe im Dienste des Klassenkampfes einzusetzen. Vor allem das Strafrecht findet hier ein weites Anwendungsfeld.

Erst dieses ständige Definieren der Klassenlage und der permanente Kampf um die Aufhebung der dabei ewig neu entstehenden Widersprüche treibt die rechtliche Entwicklung voran. Dem „Kampf ums Recht“ kommt damit - dialektisch gesehen - eine beinahe prometheische Rolle zu.

117 In seiner Widerspruchsrede von 1957 gibt MTt sechs Kriterien für die Unterscheidung von Richtig und Falsch, und damit $\mathrm{zwischen} \mathrm{"Freund"} \mathrm{und} \mathrm{"Feind"} \mathrm{an:}$

a) alles was gesagt und getan wird, soll der Einigung aller Nationalitäten im Land dienen und nicht der Spaltung.

b) es soli der sozialistischen Umgestaltung und dem sozialistischen Aufbau dienen und sie nicht schädigen.

c) es soll der Festigung der volksdemokratischen Diktatur dienen und sie nicht zerstören oder schwächen.

d) es soll die Führung der KP festigen helfen und sie nicht beseitigen oder schwächen wollen.

e) es soll der internationalen Solidarität des Sozialismus und aller friedliebender Völker in der Welt dienen und sie nicht schädigen (vgl. T. Grimm, a. a. O., S. 99 f.). 


\section{Zusammenfassung}

Die theoretischen Richtlinien, denen das chinesische Gesetzgebungsverfahren unterliegen sollte, haben sich in der Praxis nicht durchsetzen können, wie insbesondere die fünf Stadien der Legislativgeschichte beweisen.

Die "Gesetzgebungs"politik blieb vielmehr völlig im Sog der allgemeinen politischen Entwicklung und pendelte dabei in Extrempositionen ein, die bisweilen so weit auseinanderliegen, daß eine gemeinsame Rechtslandschaft fast nicht mehr auszumachen ist. Wie kraß solche Unterschiede ausfallen können, beweist insbesondere ein Vergleich zwischen der "westlichen“ Legislaturperiode 1954/55 und der ganz im Zeichen des Maoismus stehenden Epoche 1966/67. Wurden dort die "Gesetze" noch fast ausschließlich von Staatsorganen erlassen und mit soliden juristischen Methoden erarbeitet, so ist hier beinahe die gesamte Legislative auf das ZK und seine Ausschüsse übergegangen, wobei freilich nur noch Rahmenanweisungen ergehen, deren Rechtscharakter nicht immer einwandfrei zu identifizieren ist. Dies gilt in besonderem Maße von den „jüngsten Weisungen des Vorsitzenden Mao Tse-tung", die vor allem dessen "charismatisches" Herrschaftskonzept erkennen lassen. Beide Extremzustände zeigen etwa die äußersten Konturen jenes Rahmens auf, innerhalb dessen die chinesische Gesetzgebung wohl auch in $\mathrm{Zu}$ kunft funktionieren wird. Die maoistischen Elemente, die vermutlich auch in den nächsten Jahren noch eng mit der Legislative verbunden bleiben, lassen sich mit der triadischen Formel „Massenlinie, Praxisdogma und Widerspruchstheorie“ zusammenfassen.

Das Postulat der "Massenlinie“ wirkt sich dahin aus, daß die fast nur noch als Rahmennormen erlassenen "Gesetze“ erst bei ihrer Aktualisierung durch die "Volksmassen" volle Wirkung entfalten. Entelechial rücken also "Gesetz"gebung und "Gesetzes"anwendung aufs engste zusammen. Leitlinie für die Ausfüllung der überdimensionalen Rechtslücken bilden die "Ideen Mao Tse-tungs“. Das alte Tauziehen zwischen "li“ (Sitte) und „fa“ (positives Recht) taucht damit in moderner Version wieder auf. Auch das Praxisdogma lenkt die Aufmerksamkeit mehr auf die Anwendung als auf die Normschöpfung hin: Ist nämlich die Praxis das entscheidende Kriterium für die „Richtigkeit“ von Rechtsbestimmungen, so kommt es weniger auf juristisch einwandfreie Gesetze als vielmehr auf die Evolution der Gesetzlichkeit im Vollzuge an. Kraft der „schöpferischen Spontaneität der Volksmassen " ist sichergestellt, daß die dialektische Selbstentfaltung des „Richtigen“ im Wege der Praxis sich korrekt vollzieht. In Bewegung gehalten wird dieser Prozeß der Selbstentfaltung durch permanente Klassenanalyse, deren diagnostisch ermittelte Widersprüche kontinuierlich durch die Therapie des Klassenkampfes gelöst und auf eine immer höhere "juristische" Ebene angehoben werden. Fertige Gesetze könnten diesen Prozeß allenfalls hemmen. 